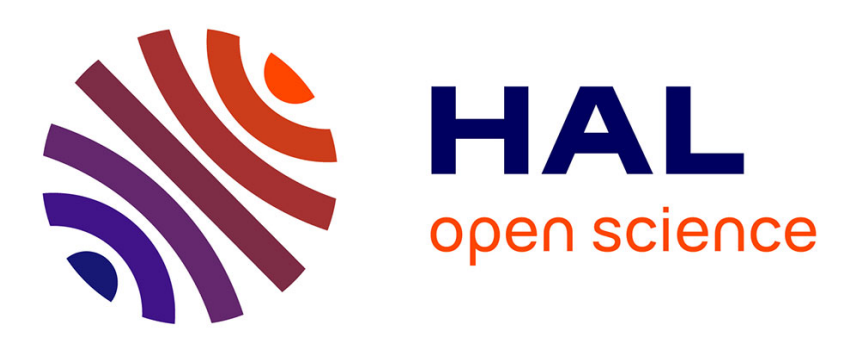

\title{
Modeling of a New Electron Acceleration Mechanism Ahead of Streamers
}

\author{
Kevin M. A. Ihaddadene, Joseph R. Dwyer, Ningyu Liu, Sébastien Celestin, \\ Feng Shi
}

\section{- To cite this version:}

Kevin M. A. Ihaddadene, Joseph R. Dwyer, Ningyu Liu, Sébastien Celestin, Feng Shi. Modeling of a New Electron Acceleration Mechanism Ahead of Streamers. Journal of Geophysical Research Space Physics, 2019, 10.1029/2018JA026084 。 insu-02263528

\section{HAL Id: insu-02263528 https://hal-insu.archives-ouvertes.fr/insu-02263528}

Submitted on 26 Aug 2019

HAL is a multi-disciplinary open access archive for the deposit and dissemination of scientific research documents, whether they are published or not. The documents may come from teaching and research institutions in France or abroad, or from public or private research centers.
L'archive ouverte pluridisciplinaire HAL, est destinée au dépôt et à la diffusion de documents scientifiques de niveau recherche, publiés ou non, émanant des établissements d'enseignement et de recherche français ou étrangers, des laboratoires publics ou privés. 


\section{JGR Space Physics}

\author{
RESEARCH ARTICLE \\ 10.1029/2018JA026084 \\ Key Points: \\ - Collisions between large negative \\ streamers and small neutral \\ plasmapatches produce thermal \\ runaway electrons \\ - Single streamer-streamer \\ head-on collision and multiple \\ streamer-streamer head-on collisions \\ are unlikely sources of runaway \\ electrons \\ - X-ray emissions by laboratory spark \\ discharges are unlikely to occur at \\ the first burst of streamers
}

Supporting Information:

- Supporting Information S1

- Movie S1

Correspondence to:

K. M. A. Ihaddadene,

kevin.ihaddadene@unh.edu

Citation:

Ihaddadene, K. M. A., Dwyer, J. R., Liu, N., Celestin, S., \& Shi, F. (2019) Modeling of a new electron acceleration mechanism ahead of streamers. Journal of Geophysical Research: Space Physics, 124. https:// doi.org/10.1029/2018JA026084

Received 12 SEP 2018

Accepted 24 JUL 2019

Accepted article online 4 AUG 2019

C 2019. American Geophysical Union. All Rights Reserved.

\section{Modeling of a New Electron Acceleration Mechanism Ahead of Streamers}

\author{
Kevin M. A. Ihaddadene ${ }^{1}(\mathbb{D})$, Joseph R. Dwyer ${ }^{(\mathbb{D}}$, Ningyu Liu' ${ }^{1} \mathbb{D}$, Sebastien Celestin ${ }^{2} \mathbb{D}$, \\ and Feng Shi ${ }^{3}$ \\ ${ }^{1}$ Space Science Center, University of New Hampshire, Durham, NH, USA, ${ }^{2}$ LPC2E, University of Orleans, CNRS, \\ Orleans, France, ${ }^{3}$ Department of Physics, Auburn University, Auburn, AL, USA
}

\begin{abstract}
Head-on collisions between negative and positive streamers have been proposed as a mechanism behind X-ray emissions by laboratory spark discharges. Recent simulations using plasma fluid and particle in cell models of a single head-on collision of two streamers of opposite polarities in ground pressure air predicted an insignificant number of thermal runaway electrons $>1 \mathrm{keV}$ and hence weak undetectable X-ray emissions. Because the current available models of a single streamer collision failed to explain the observations, we first use a Monte Carlo model coupled with multiple static dielectric ellipsoids immersed in a subbreakdown ambient electric field as a description of multiple streamer environment and we investigate the ability of multiple streamer-streamer head-on collisions to accelerate runaway electrons $>1 \mathrm{keV}$ up to energies $\sim 200-300 \mathrm{keV}$ instead of just one single head-on collision. The results of simulations show that the streamer head-on collision mechanism fails to accelerate electrons; instead, they decelerate in the positive streamer channel. In a second part, we use a streamer plasma fluid model to simulate a new streamer-electron acceleration mechanism based on a collision of a large negative streamer with a small neutral plasma patch in different Laplacian electric fields $\left|E_{0}\right|=(35,40,45) \mathrm{kV} / \mathrm{cm}$, respectively. We observe the formation of a secondary short propagating negative streamer with a strong peak electric field $>250$ up to $378 \mathrm{kV} / \mathrm{cm}$ over a time duration of $\sim 0.16 \mathrm{~ns}$ at the moment of the collision. The mechanism produces up to $10^{6}$ runaway electrons with an upper energy limit of $24 \mathrm{keV}$.
\end{abstract}

\section{Introduction}

The mechanisms behind the production of runaway electrons, $\mathrm{X}$ - and gamma-rays by laboratory spark discharges, lightning leaders, and thunderstorms are still not yet understood. How such runaway electrons are produced is a key question in the field of atmospheric and space electricity. We know that negative lightning stepped leaders produce bursts of X-rays (Dwyer et al., 2003; Dwyer, Rassoul, Al-Dayeh et al., 2005; Moore et al., 2001), and many observation campaigns have been made to understand those emissions (e.g., Dwyer et al., 2011; Howard et al., 2008; Saleh et al., 2009; Schaal et al., 2014). The observations of X-rays were numerically analyzed by Babich et al. (2013). Laboratory spark discharges ignited by megavolt Marx generators produce X-rays as well (Dwyer, Rassoul, Saleh et al., 2005; Kochkin et al., 2012, 2015; March \& Montanyà, 2011; Nguyen et al., 2008, 2010; Rahman et al., 2008). The presence of plasma streamer filaments in both lightning leader tips and laboratory spark discharges leads to the suggestion that streamers are behind those high-energy radiations, since streamers carry strong peak electric fields at their tips that may reach values higher than $\sim 270 \mathrm{kV} / \mathrm{cm}$ at ground pressure under some circumstances. The value of $270 \mathrm{kV} / \mathrm{cm}$ is a deterministic electric field (e.g., Babich \& Stankevich, 1973; Babich, 2003; Green \& Peterson, 1968; Moss et al., 2006; Peterson \& Green, 1968) necessary to accelerate the thermal low-energy electrons (tens of electron volts, e.g., Eichwald et al., 2006) present in the streamer head to high energies $>120 \mathrm{eV}$. At this field the electric force exceeds the friction force caused by air molecules, resulting in so-called thermal runaway electron acceleration. The investigation of X-rays from laboratory sparks and explosive emission of electrons in vacuum have been a focus of many groups for many years with a significant body of literature published so far (e.g., Aleksandrov, 1966; Babich et al., 1990; Babich, 2005; Babich \& Stankevich, 1973; Borukhov et al., 1973; Bugaev et al., 1975; Gurevich, 1961; Kremnev \& Kurbatov, 1972; Litvinov et al., 1983; Mesyats et al., 1972; Stankevich \& Kalinin, 1968; Stankevich, 1971; Tarasova \& Khudyakova, 1970). Understanding the high-energy emissions from both lightning and laboratory sparks may be important for understanding terrestrial gamma ray flashes, which is the main focus of the recently launched ASIM 
instrument (Neubert, 2009; Neubert et al., 2019) to the International Space Station, or missions under preparation such as the TARANIS satellite (Lefeuvre et al., 2008).

One of the mechanisms suggested to produce thermal runaway electrons is the head-on collision between negative and positive streamers, first proposed by Cooray et al. (2009). Head-on collisions may happen frequently in a laboratory spark discharge and during the connection between a main negative lightning leader and a bidirectional space leader. Cooray et al. (2009) used an electrostatic approach to study the problem of head-on collisions between a large number of positive and negative streamers. Processes associated with the dynamics of the streamers, ionization, attachment, conductivity, and relaxation were not considered in their study. Ihaddadene and Celestin (2015) reported the first numerical study of the production of X-rays by a single streamer head-on collision in a small gap of $8 \mathrm{~mm}$, in an ambient field up to $60 \mathrm{kV} / \mathrm{cm}$ using a streamer fluid model and showed a significant increase of the electric field $(\sim 267 \mathrm{kV} / \mathrm{cm})$ as the positive and negative streamer heads neutralize each other. However, such a high field exists over a very short time scale $<10$ ps. They showed a production of maximum number of 2,000 thermal runaway electrons $>1 \mathrm{keV}$ per single streamer collision. The 2,000 runaway electrons were estimated in two separate steps. They first modeled the increase of the electric field in a streamer head-on collision between negative and positive streamers, then they used the time-varying electric field $\left(E_{\max }>250 \mathrm{kV} / \mathrm{cm}\right)$ in a Monte Carlo simulation as a homogeneously distributed electric field in space to maximize the production of thermal runaway electrons. Both the number and the energy of the runaway electrons were insufficient to explain the energy of the X-rays (50-500 keV) observed in the experiments (Dwyer, Rassoul, Saleh et al., 2005; Dwyer, Rassoul, Al-Dayeh et al., 2005). Kochkin, Köhn, et al., (2016) estimated a necessary number of $10^{6}$ runaway electrons from head-on collisions with energies $\sim 100 \mathrm{keV}$ per one electron to explain the observations of $10^{4} \mathrm{X}$-ray photons in a laboratory experiment. A criticism was given when the results of Ihaddadene and Celestin (2014) were first presented to the scientific community because the potential difference used $(60 \mathrm{kV} / \mathrm{cm} \times$ $0.8 \mathrm{~cm}=48 \mathrm{kV}$ ) was not large enough to accelerate electrons to high energies compared to the $1 \mathrm{MV}$ potential difference available in laboratory long gap experiments. In addition, the Ihaddadene and Celestin (2015) streamer head-on collision and the runaway electrons simulations were not self-consistent and the parallel plates boundary conditions were not a perfect representation of laboratory experimental conditions. Babich and Bochkov (2017) simulated a streamer head-on collision in a 5-cm gap with a $250-\mathrm{kV}$ potential difference and they found similar conclusions as in Ihaddadene and Celestin (2015). Babich and Bochkov (2017) also used electron transport coefficient derived using the BOLSIG+ code (Hagelaar \& Pitchford, 2005) instead of Morrow and Lowke (1997) along with open boundary conditions, and the effects did not change the conclusions of Ihaddadene and Celestin (2015). Moreover, da Silva et al. (2017, and references therein) detected $\mathrm{X}$-ray photons ranging from 33 to $96 \mathrm{keV}$ in a 4- to 8-cm short gap experiment with a lower applied voltage of $100 \mathrm{kV}$. The streamers they observed were $1-\mathrm{cm}$ long and the X-rays were not associated with any streamer head-on collision (see also Kochkin et al., 2015, section 3.5.2). The X-ray bursts observed in long gap experiment are more intense than those observed in a short gap experiment presumably because the electrons experience a larger potential difference when they cross the gap (e.g., Kochkin et al., 2012, 2015; da Silva et al., 2017). Köhn et al. (2017) used full self-consistent particle in cell (PIC) simulations to investigate the acceleration of electrons during a single streamer head-on collision and they found no more than one electron with energy $>500 \mathrm{eV}$ was produced. They drew the same conclusions as in Ihaddadene and Celestin (2015). Luque (2017) investigated the radio emissions from a single streamer head-on collision in long gap up to $30 \mathrm{~cm}$ using a full Maxwell's equations model in a protrusion-protrusion geometry and an applied electric potential of $750 \mathrm{kV}$. To estimate the energy of runaway electrons he used the one-dimensional deterministic equation for the acceleration of a single test electron in a homogeneous electric field. He assumed an already produced 1-keV electron in the high field zone and found a gain after the acceleration in few millimeters to $2 \mathrm{~cm}$ of $5 \mathrm{keV}$ and $100 \mathrm{keV}$, respectively. The acceleration of an electron in a time-varying electric field (collapse) is different from that in a static electric field profile pulse because of the very short duration of the streamer head-on collision (picosecond).

Another mechanism that may produce thermal runaway electrons is the process of streamer branching (Arrayás et al., 2002; Kulikovsky, 2000, 2001, 2002; Liu \& Pasko, 2004; Moss et al., 2006; Pancheshnyi \& Starikovskii, 2001; Rocco et al., 2002). Prior to the branching the streamer radius expands and reaches its maximum value of $\sim 0.2-0.4 \mathrm{~cm}$ with an accumulated potential drop of $\sim 100 \mathrm{kV}$ (e.g., Celestin \& Pasko, 2011). As a streamer approaches its maximum radius, the peak electric field in the streamer head is expected to increase in order to keep the preionization ahead of the streamer head on a similar level 
(Liu \& Pasko, 2004, sections 4.1 and 4.4). This may result in a peak streamer field $>250 \mathrm{kV} / \mathrm{cm}$. However, up to the present time no full physical 3-D simulation of streamer branching has been conducted to verify such expected streamer behavior, even though there have been great advances in 3-D streamer simulations in recent years (e.g., Li et al., 2009; Papageorgiou et al., 2011; Plewa et al., 2018; Shi et al., 2017; Teunissen \& Ebert, 2017). This branching hypothesis has been assumed in Moss et al. (2006) to justify the high electric field used in their Monte Carlo simulations to study the production of thermal runaway electrons by a negative streamer. Moss et al. (2006) used a $\sim 300-\mathrm{kV} / \mathrm{cm}$ square pulse electric field in various ambient electric fields $\sim 75 \mathrm{kV} / \mathrm{cm}$ to $\sim 150 \mathrm{kV} / \mathrm{cm}$. However, the description of a streamer by a square pulse in ambient electric fields higher than what may exist in a leader tip ( $\sim 50 \mathrm{kV} / \mathrm{cm}$; Raizer, 1991, p.363) may overestimate the energy and the flux of runaway electrons. Later on, Celestin and Pasko (2011) came up with a refined electric field shape of a streamer where a streamer simulation in an ambient electric field of $50 \mathrm{kV} / \mathrm{cm}$ was used. They estimated analytically the streamer parameters prior to the branching such as the radius and the potential drop, and then they injected in a Monte Carlo simulation the streamer electric field multiplied by a factor $\sim 1.5$ to reach a peak field of $\sim 270 \mathrm{kV} / \mathrm{cm}$ required for the production of thermal runaway electrons. They accelerated the electrons in a maximum available potential difference in the streamer head of $\sim 100$ $\mathrm{kV}$ as they deduced from their analytical calculations. Celestin and Pasko (2011, section 4) came up with a runaway electron average energy value of $\sim 60 \mathrm{keV}$.

Chanrion and Neubert (2010) proposed a single negative streamer mechanism that accelerates thermal electrons to explain terrestrial gamma ray flash observations. Their work is based on calculations by an advanced PIC-MCC code with an adaptive resampling scheme, the stochastic probability for an electron to runaway in a homogeneous electric field. They used that probability in a streamer PIC model to estimate in a stochastic manner the production of thermal runaway electrons. The probability was estimated in a homogeneous electric field that was not affected by the space charge. Based on their analysis, for every electric field value there is a certain probability for an electron to run away. The higher the number of available thermal electrons is, the higher the number of runaway electrons is generated $N_{e} \times P_{e}(E)=N_{\text {run }}$. For $E \geq 9 E_{k}=288 \mathrm{kV} / \mathrm{cm}$, where $E_{k}$ is the breakdown electric field, the probability is equal to one. Because of the computational cost their streamer model does not include the photoionization, which is known to affect the propagation of both negative and positive streamers (e.g., Kulikovsky, 2001, 2002; Liu \& Pasko, 2004). Moreover, they used a background electric field up to $\sim 100 \mathrm{kV} / \mathrm{cm}$ and a negative streamer peak electric field reached a value of $\sim 320 \mathrm{kV} / \mathrm{cm}$. When high ambient electric fields $100-1,000 \mathrm{kV} / \mathrm{cm}$ are used in experiments, a homogeneous ionized background develops and dominates the streamer regime (e.g., Mesyats \& Bychkov, 1968). The first runaway electron with energy up to $171 \mathrm{keV}$ appeared when the electric field peaked at $\sim 157 \mathrm{kV} / \mathrm{cm}$ (Chanrion \& Neubert, 2010, see Figure 3) instead of the deterministic value of $\sim 250 \mathrm{kV} / \mathrm{cm}$. From the point of view of the peak electric field the deterministic approach constrains more the ignition of the first population of thermal runaway electrons compared to the stochastic approach. For the case of a positive streamer, the acceleration is inefficient even though the peak electric field is higher than the negative one because the electrons flow into the weak field of the positive streamer body (Chanrion \& Neubert, 2010, para. 34).

Ihaddadene (2016, pp.101-114) used a streamer plasma fluid model to reproduce the experimental results of Kremnev and Kurbatov (1972). Thermal runaway electrons and associated X-rays were produced by a single negative streamer in a 4-mm gap and a high ambient electric field $50 \mathrm{kV} / \mathrm{cm}$ at a standard pressure air (76-760 torr). Ihaddadene (2016) showed a production of runaway electrons between 1 and $6 \mathrm{keV}$ and $\mathrm{X}$-ray total energy between 0.00125 and $0.26 \mathrm{~nJ}$ in an ambient air density variation of $50 \%$ to $80 \%$, respectively. They found a reasonable agreement between modeling and experiment. Recently, Köhn, Chanrion, Babich, et al. (2018) used a PIC code to simulate a double-headed streamer in a similar ambient field and size gap subjected to spatial sinusoidal air density variations along $r$ and $z$ axes between $15 \%$ and $80 \%$ to study the properties of streamers. Their simulation results showed the production of electron patches at the front of the main positive streamer possibly due to the photoionization. They stated that those patches acted as ignition points for negative streamers that collided afterward with the main positive streamer and then produced runaway electrons up to $3 \mathrm{keV}$ in the $80 \%$ ambient air density variation case. The electron patches could be similar to the known stem structures or pilot systems usually observed in laboratory spark experiments described by, e.g., Kochkin et al. (2015) and Reess et al. (1995). However, as the model is a 2.5 Monte Carlo code, the patches have a cylindrical shape. Reducing the air density and keeping the same applied field is by definition an increase of the reduced electric field $\frac{E}{N}$ and equivalent to high ambient fields that were used by, e.g., Chanrion and Neubert (2010), Moss et al. (2006), and Ihaddadene (2016, pp.101-114). 


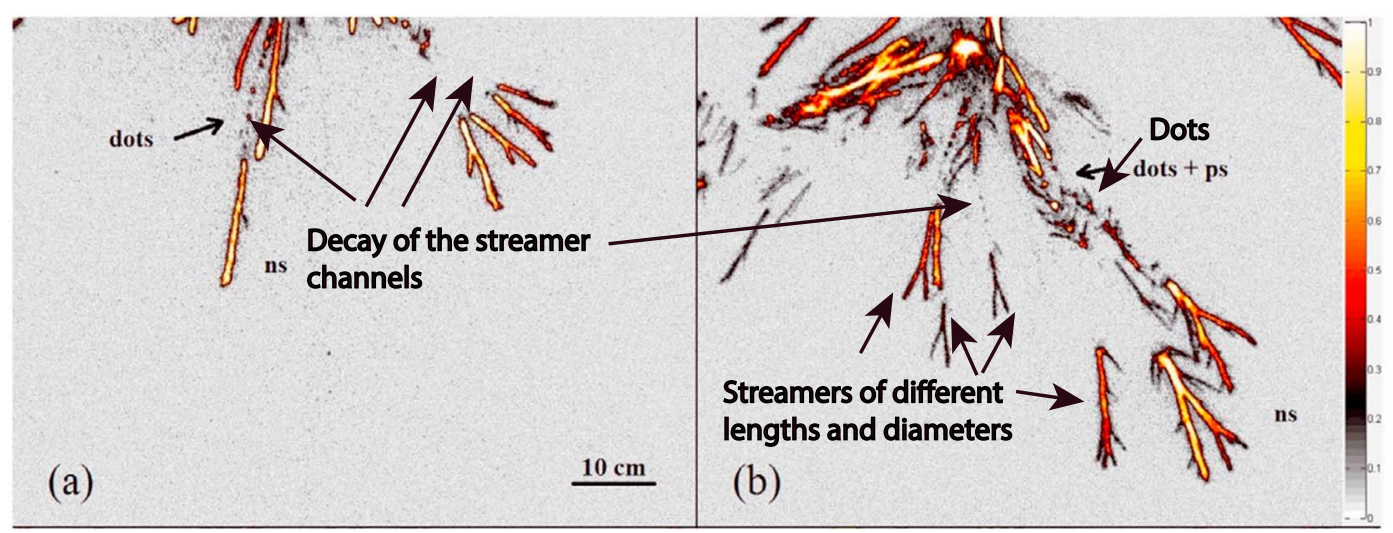

Figure 1. (a)-(b) Multiple visible dots, short and long streamers, streamer branching, and the complex morphology of discharge plasmas in a laboratory spark discharge. Adapted from (Kochkin et al., 2014).

Interestingly, if low ambient air density pockets occur in laboratory sparks, this indicates that the head-on collision is not required and a single streamer alone could produce thermal runaway electrons. It would be interesting to quantify at which value of low electric field and minimum density change the X-rays can be produced. Recently, Köhn, Chanrion and Neubert (2018) made a focused study on high-energy radiation from streamers and concluded that air density perturbations may be a candidate for the acceleration of runaway electrons.

As a single-streamer head-on collision poorly describes laboratory spark discharges because of the large number of streamers present (see Figure 1), in this work we investigate the multiple-streamer head-on collisions problem and the motion of a negative streamer into a neutral plasma patch. In the first part, we use a uniform distribution of multiple static dielectric ellipsoids as a streamer-like representation of the electric field in a Monte Carlo model to search for the configuration that efficiently accelerates the electrons. In the second part, we use a streamer plasma fluid model and simulate a new streamer-electron acceleration mechanism based on the collision between a large negative streamer and a small neutral plasma patch (see Figure 2). We will show that this mechanism is more efficient to accelerate electrons than streamer-streamer head-on collision.

\section{Model Formulation}

\subsection{Monte Carlo Dielectric Ellipsoids Model}

The Monte Carlo model we use in the present study is the 3-D runaway electron avalanche model that simulates the physics of runaway electron avalanche from $\sim 1 \mathrm{keV}$ to several hundred megaelectron volts. The model is fully described by Dwyer $(2007,2012)$. To study the dynamics of runaway electrons in a streamer head-on collision we implement in a Monte Carlo model an inhomogeneous electric field profile generated by one, two, or more static dielectric ellipsoids embedded in a homogeneous ambient electric field $E_{0}$. The electrostatic analytic solution of the ellipsoid electric potential is well known (e.g., Landau \& Lifshitz, 1960; Stratton, 1941, pp.207-217, pp.19-42), and we derive the electric field inside and outside the ellipsoid by taking the gradient of the following equations (1) and (2), respectively:

$$
\phi=-\frac{E_{0} z}{1+\left(\frac{a b c}{2}\right)\left(\frac{\epsilon_{1}-\epsilon_{2}}{\epsilon_{2}}\right) \int_{0}^{\infty} \frac{\mathrm{d} s}{\left(s+a^{2}\right) R_{s}}}
$$

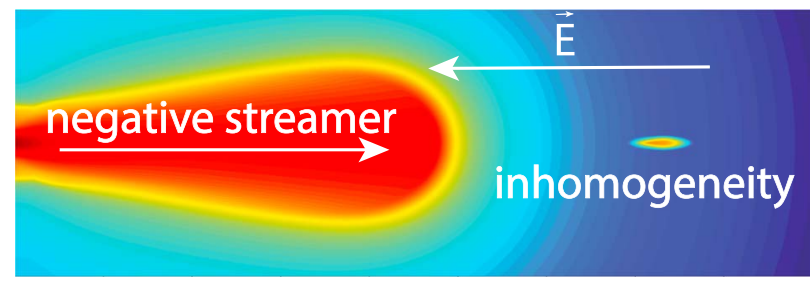

Figure 2. Illustration of a collision between a negative streamer (left) and a small plasma patch (right) in an external electric field $\vec{E}$. 


$$
\phi=\phi_{0}\left(1-\frac{\left(\frac{a b c}{2}\right)\left(\frac{\epsilon_{1}-\epsilon_{2}}{\epsilon_{2}}\right) \int_{\zeta}^{\infty} \frac{\mathrm{d} s}{\left(s+a^{2}\right) R_{s}}}{1+\left(\frac{a b c}{2}\right)\left(\frac{\epsilon_{1}-\epsilon_{2}}{\epsilon_{2}}\right) \int_{0}^{\infty} \frac{\mathrm{d} s}{\left(s+a^{2}\right) R_{s}}}\right)
$$

where $\phi_{0}=-E_{0} z, \quad R_{s}=\left(s+a^{2}\right)^{\frac{1}{2}}\left(s+b^{2}\right)^{\frac{1}{2}}\left(s+c^{2}\right)^{\frac{1}{2}}, \quad \zeta=\frac{1}{4}\left(r_{1}+r_{2}\right)^{2}-a^{2}, r_{1}=$ $\left(\left(x-x_{0}\right)^{2}+\left(y-y_{0}\right)^{2}+\left(z-z_{0}-a e\right)^{2}\right)^{\frac{1}{2}}, r_{2}=\left(\left(x-x_{0}\right)^{2}+\left(y-y_{0}\right)^{2}+\left(z-z_{0}+a e\right)^{2}\right)^{\frac{1}{2}}$, and $e=\left(1-\frac{b^{2}}{a^{2}}\right)^{\frac{1}{2}}$. The parameters $a, b$, and $c$ are the semiprincipal axis of the ellipsoid along $\vec{e}_{z}, \vec{e}_{x}$, and $\vec{e}_{y} \operatorname{directions;} r_{1}$ and $r_{2}$ are the distances of a given point to the foci of the ellipsoid and $e$ its eccentricity. The coordinates $(x, y, z)$ and $\left(x_{0}, y_{0}, z_{0}\right)$ correspond to the positions of the electron and the ellipsoid, respectively. Based on different ellipsoid sizes, between 4-mm and 14-cm long, extremities of two ellipsoids separated by 1 to $4 \mathrm{~mm}$ along the $z$ direction, and parallel ellipsoids by 10 to $30 \mathrm{~mm}$ along the $x$ direction, in ambient electric fields $E_{0}=10-30 \mathrm{kV} / \mathrm{cm}$, we test whether or not the runaway electrons continue to accelerate. We position the electrons in different locations between the ellipsoid heads, in the middle of the ellipsoid channel equivalently to an already collapsed collision, and in the background field in between the ellipsoid bodies. We calculate analytically the corresponding electric field at a given location of the electron $(x, y, z)$. In this study we take $c=b$. The parameter $\epsilon_{1}$ is the permittivity of the ellipsoid that is embedded in a homogeneous medium of inductive capacity $\epsilon_{2}$, which is the air in this case. Equivalently to the streamer channel, $\epsilon_{1}$ is chosen to adjust the value of the electric field inside the ellipsoid body to approximate the streamer channel.

\subsection{Negative Streamer and Plasma Patch Model}

To study the collision between an expanding negative streamer and a small neutral plasma patch, we use the streamer model based on the drift-diffusion equations for charged species coupled with Poisson's equation and which is fully described in Ihaddadene and Celestin $(2015,2017)$.

We choose to use a sphere-to-plane electrode configuration (e.g., Babaeva \& Naidis, 1996a; 1996b) to initiate a single negative streamer connected to a point electrode with open boundary conditions. To obtain a maximum amplitude of the electric field of $\left|\sim 4 E_{k}\right|$ at the surface of the sphere $\left(E_{\mathrm{sph}}=\frac{\phi_{0}+3 E_{0} R}{R}\right)$ (e.g., Liu et al., 2006, 2009) a sphere electrode of a radius $R_{\mathrm{sph}}=10^{-3} \mathrm{~m}$ is set to a potential $\phi_{0}=-1.5,0$, and $1.5 \mathrm{kV}$ and placed in uniform electric fields $E_{0}=-35,-40$, and $-45 \mathrm{kV} / \mathrm{cm}$, respectively. The value of the breakdown electric field $E_{k}=28.7 \mathrm{kV} / \mathrm{cm}$ is calculated based on Morrow's coefficients (Morrow \& Lowke, 1997). We have to mention that Morrow's coefficients underestimate the ionization frequency with respect to BOLSIG+ coefficients as demonstrated by Babich and Bochkov (2017, see Figure 1) and so one expects higher streamer electric fields than the results presented in this paper if one uses BOLSIG+ coefficients. In this study, we consider $E_{0}=-35$ and $E_{0}=-45 \mathrm{kV} / \mathrm{cm}$ as lower and upper ambient electric fields. These electric field amplitudes are widely used in the streamer literature to describe the strength of the field in the streamer ignition zone in laboratory spark discharges and lightning leader tips (e.g., Babich et al., 2015; Babich \& Bochkov, 2017; Bazelyan \& Raizer, 1998; Rakov \& Raizer, 2000; Chanrion \& Neubert, 2010; Celestin \& Pasko, 2011; Ihaddadene \& Celestin, 2015; Köhn et al., 2017p. 76-84,p. 62-74). One case with a sphere of a radius $R_{\mathrm{sph}}=10^{-2} \mathrm{~m}, \phi_{0}=-6.5 \mathrm{kV}$, and $E_{0}=-28 \mathrm{kV} / \mathrm{cm}$ over a short gap of $8 \mathrm{~mm}$ is also presented.

The negative propagating streamer is initiated by placing a Gaussian $n_{e}=$ $n_{e_{0}} \exp \left(-\left(z-2 \sigma_{z}\right)^{2} / \sigma_{z}^{2}\right) \exp \left(-r^{2} / \sigma_{r}^{2}\right)$ of neutral plasma cloud with characteristic sizes $\sigma_{z}=2 \times 10^{-4} \mathrm{~m}, \sigma_{r}=$ $2 \times 10^{-4} \mathrm{~m}$, and $n_{e_{0}}=10^{20} \mathrm{~m}^{-3}$ near the vicinity of the sphere electrode. The size of the simulation domain varies from $1,001 \times 377$ to $2,625 \times 377$ regular grid points along $z$ and $r$ axis, respectively, with a spatial resolution defined by $\Delta z=\Delta r=8 \times 10^{-6} \mathrm{~m}$. The dimensions of the Gaussian neutral plasma patch are $\sigma_{z}=2 \times 10^{-4} \mathrm{~m}, \sigma_{r}=15 \times 10^{-6} \mathrm{~m}$, and $n_{e_{0}}=10^{18} \mathrm{~m}^{-3}$, and it is located at a distance $d_{\text {patch }}$ from the sphere electrode.

We choose to use a weak peak electron density $n_{e_{0}}=10^{18} \mathrm{~m}^{-3}$ to prevent the initiation of streamers from the plasma patch and avoid conventional streamer-streamer collision. The patch is positioned at different locations $d_{\text {patch }}=(0.5,1,1.4) \mathrm{cm}$ in -40 and in $-45 \mathrm{kV} / \mathrm{cm}$, at $(0.5,1.4) \mathrm{cm}$ in $-35 \mathrm{kV} / \mathrm{cm}$, and at $0.5 \mathrm{~cm}$ in $-28 \mathrm{kV} / \mathrm{cm}$.

\section{Results}

\subsection{Streamer-Streamer Head-On Collision}

The multistreamer discharge problem is complex to model if electromagnetic, hydrodynamic, and kinetic processes are included in one single model. In laboratory discharge experiments multiple streamers $\left(\sim 10^{6}\right.$ 

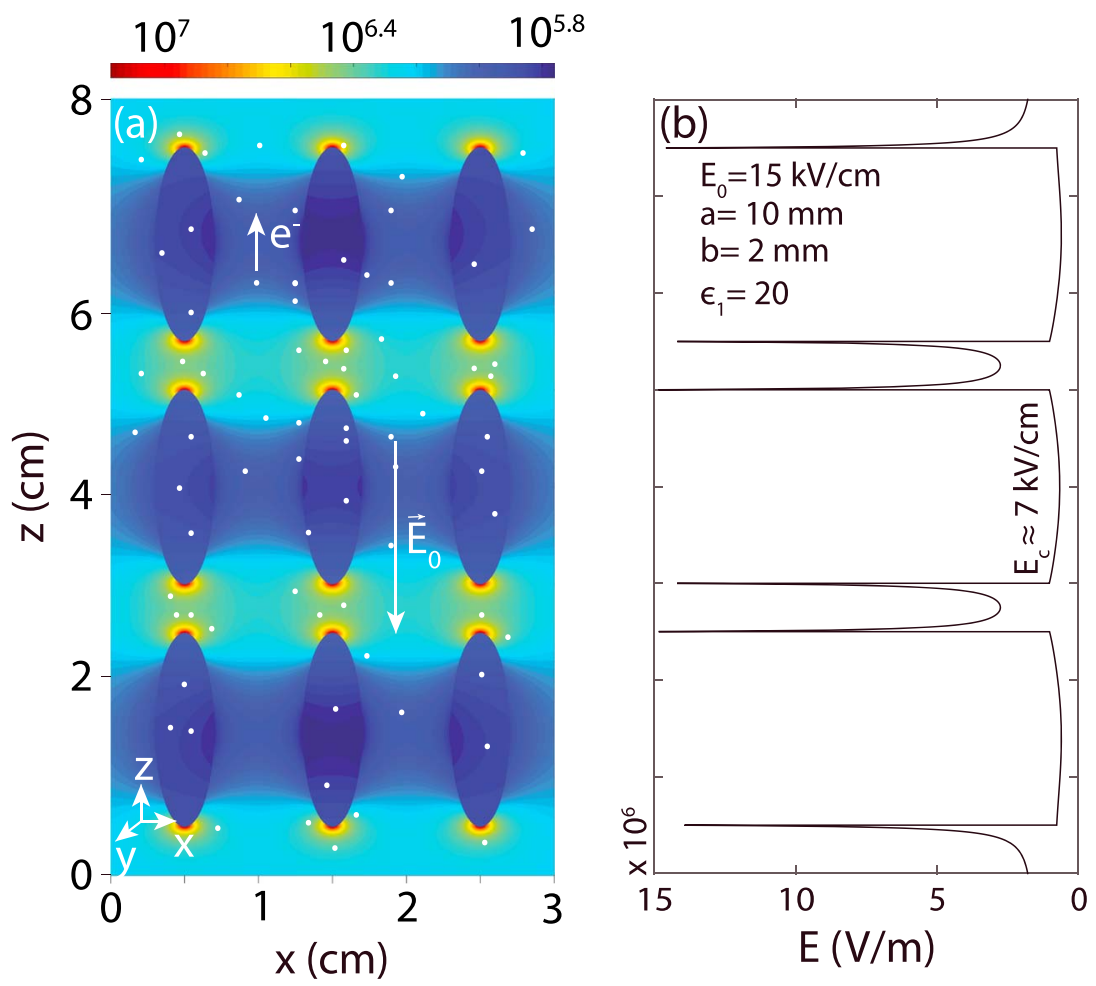

Figure 3. (a) Cross-sectional view of the electric field produced by several dielectric ellipsoids immersed in an ambient electric field $E_{0}=15 \mathrm{kV} / \mathrm{cm}$. The white dots illustrate electrons moving in between the ellipsoids. (b) Electric profile along the $z$ axis (one streamer column).

Bazelyan \& Raizer, 1998, section 2.4) with high localized electric fields are distributed inhomogeneously (see Figure 1) where the electric charge is a conserved quantity. From previous PIC simulations of a single-streamer head-on collision in air at ground pressure, results seem unlikely to explain the observations of X-ray emissions, with no more than one electron of $500 \mathrm{eV}$ produced under an ambient electric field of $60 \mathrm{kV} / \mathrm{cm}$ (Köhn et al., 2017). In this work, we explore whether multiple streamers combined with multiple head-on collisions can possibly accelerate the electrons $(\geq 1 \mathrm{keV})$ produced by a single-streamer head-on collision (Ihaddadene \& Celestin, 2015; Köhn et al., 2017) to the higher values (50-500 keV) observed in the experiments. The hypothesis is that the electrons produced in the collision zone could cross the body of the positive streamer, reach another negative streamer head, then another positive streamer head, or another streamer head-on collision, and so on until finally the electrons would gain enough energy from multiple streamers to run away in a discharge gap. To test this hypothesis, we use a static distribution of multiple dielectric ellipsoids of different lengths and diameters equivalent in size to streamers we usually observe in laboratory experiments (see Figure 3) with peak and body electric fields reasonably close to streamer simulation results. Similar modeling approaches and different electric field configurations have been previously used to study runaway electron production (e.g., Köhn \& Ebert, 2015; Kochkin, Köhn, et al., 2016; Kochkin, Lehtinen, et al., 2016). For an average applied electric field over a certain length the available potential difference must be conserved and the introduction of a dielectric ellipsoid approach is a good approximation for the multistreamer problem, as it modifies the uniform electric field to high and low field zones without introducing additional external charge or affecting the available potential difference. The ellipsoids are a simplified representation of a collection of streamers occurring in a laboratory discharge. The well-known analytic solution (equations (1) and (2)) makes it easy to implement in a Monte Carlo model.

Figure 3a shows the cross-sectional view of one of the configurations of multiple dielectric ellipsoids immersed in an ambient field $E_{0}=15 \mathrm{kV} / \mathrm{cm}$ close to the average experimental value $\frac{1}{1} \mathrm{MV} / \mathrm{m}=10 \mathrm{kV} / \mathrm{cm}$. One can see the encounter of multiple ellipsoid heads forming multiple high field zones similar to streamer head-on collisions where runaway electrons can be produced. Based on the orientation of the ambient electric field, the upward and downward ellipsoid heads refer to negative and positive streamers, respectively. 


\begin{tabular}{lcc}
$\begin{array}{l}\text { Table 1 } \\
\text { Runaway Electron Energy } \varepsilon_{\text {run }} \text { Required in Different Homogeneous } \\
\text { Background Electric Fields, Deterministic (Equation (3)), and Stochastic } \\
\text { Approach }\end{array}$ & $\begin{array}{c}\text { Stochastic } \varepsilon \\
(\mathrm{keV})\end{array}$ \\
\hline E & Deterministic $\varepsilon$ & 60 \\
$(\mathrm{kV} / \mathrm{cm})$ & $(\mathrm{keV})$ & 40 \\
\hline 10 & 36 & 20 \\
20 & 15 & 18 \\
30 & 9 & 15 \\
40 & 6 & 10 \\
50 & 4 & 8 \\
60 & 3 & 6 \\
70 & 2.6 & 5 \\
80 & 2 & 3 \\
90 & 1.8 & \\
100 & 1.5 & \\
\hline
\end{tabular}

Note. REAM = 3-D runaway electron avalanche model.

From streamer simulations, we know that the positive streamer head field is higher than the negative one and inversely in their channels, but using ellipsoid approximation, we are unable to simulate this detailed property of streamers. The ellipsoid does not take into account the physical processes such as ionization, relaxation, production of electron density, or a continuous flowing charge through the channel. From Figure $3 \mathrm{~b}$ one can see the nearly homogeneous field in the body of the ellipsoid and both heads possess almost similar maximum electric field. We use the superposition principle to estimate the total electric field produced by the ellipsoids. The sum of the electric field components do affect each other slightly and that is why we see a small increase of the field inside the body and the head of the ellipsoid in the middle of the figure.

The use of a simple linear superposition is justified by the fact that it leads to a reasonable representation of the electric field in streamer heads and channels as obtained in simulations. In this context the runaway electron acceleration can be easily maximized through a reorganization of the ellipsoids. We do not claim that the electric field obtained is a valid solution to the electrostatic problem involving real dielectric ellipsoids as the effect of mutual interactions should be taken into account.

To investigate the streamer head-on collision efficiency in the multiple streamer configuration, we assume there already exist 2,000 runaway electrons $\varepsilon_{i} \geq 1$ up to $50 \mathrm{keV}$ produced by a single collision (Ihaddadene $\&$ Celestin, 2015). The assumed initial energy $\varepsilon_{i}$ is lower than the runaway threshold $\varepsilon\left(E_{0}\right)$ for an electron to run away in an ambient field $E_{0}<E_{k}$. After multiple combined tests we find that no electron was able to run away. The short ellipsoids do not provide enough potential drop in the high field region to accelerate the electrons and the long ones where the electrons gain energy in between the ellipsoid heads decelerate the electrons in the low body field of the long ellipsoid channel before they reach the other negative streamer or collision point.

A single long and large ellipsoid is more efficient than two colliding ellipsoids to accelerate a 1-keV electron produced at the tip (i.e., equivalent to a large streamer at the branching stage). Electrons run away easily ahead of a single ellipsoid in the ambient field in contrast to their deceleration in the channel field in a case of two colliding ellipsoids.

Table 1 shows the energy required for an electron to runaway in a different homogeneous electric fields established by a Monte Carlo and a one-dimension deterministic equation for a test electron $\left(m_{e} \frac{\mathrm{d} v_{e}}{\mathrm{~d} t}=q E-F\right.$. For details see section 3.3). As one can see the stochastic energy is greater than the deterministic one by up to $\sim 50 \%$ due to the angular scattering which is not included in the one-dimension equation of motion. In reality, an electron does not travel in a straight line parallel to the electric field but goes through a longer path because of the scattering and requires more energy to runaway in that field. For instance, in a $10-\mathrm{kV} / \mathrm{cm}$ streamer channel field a streamer head-on collision must produce electrons $>60 \mathrm{keV}$ in the collision zone to allow them to cross the channel. This means a potential drop $>60 \mathrm{kV}$ in a high field zone must be available; 


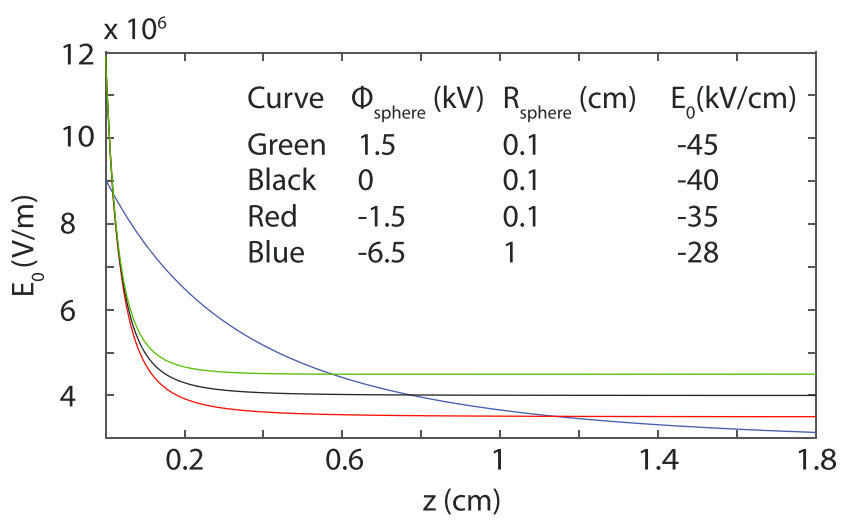

Figure 4. Ambient electric fields $E_{0}$ produced by a sphere-to-plane electrode. otherwise, the electrons will decelerate in the channel. To achieve an efficient acceleration by a conventional streamer-streamer head-on collision, a positive streamer channel field must be strong enough to accelerate the runaway electrons that are produced at the collision point $\left(\varepsilon_{\text {run }}>\varepsilon_{\mathrm{th}}^{c}\left(E_{c}\right)\right)$.

\subsection{Streamer-Patch Collision}

From the results obtained in the previous section, the conventional streamer-streamer head-on collision appears unlikely to be an efficient electron acceleration mechanism even when considering reacceleration in a multiple-streamers configuration. In this section we develop a new picture of a streamer collision that involves a large negative streamer and a neutral plasma patch or inhomogeneity seen from experiments (see Figure 1). The figure is essentially a 2-D representation of the 3-D phenomenon. Thus, even though it appears that streamers might interact with either one another or with inhomogeneities, it could be that they are separated in depth and no interaction takes place (Nijdam et al., 2008). This is an alternative solution to a conventional streamer-streamer collision channel problem as it does not involve a positive streamer but only a negative streamer moving forward with the electrons in the ambient field.

Figure 4 shows the Laplacian electric fields used in the current study. One clearly sees a highly enhanced electric field near the sphere electrode $<0.2 \mathrm{~cm}$ and homogeneous $>0.2 \mathrm{~cm}$ for the green, black, and red curves. The blue curve shows an inhomogeneous ambient field everywhere.

Figures $5 \mathrm{a}$ and $5 \mathrm{~b}$ show the cross-sectional view of the electron density and the electric field at the moment of the collision between the negative streamer and the small neutral plasma patch in case of $E_{0}=-40 \mathrm{kV} / \mathrm{cm}$. The figure shows a transition of the patch to a short negative streamer with an enhanced field at its tip due to the influence of the long negative streamer. Figures $5 \mathrm{c}$ and $5 \mathrm{~d}$ show the electron density and the electric field along the $z$ axis. One sees clearly a rightward propagating negative streamer with an unstable field and an increasing speed toward the patch. During the collision the initial electron density in the patch increases to a level that initiates a streamer because of the influence of the long streamer peak field. The charge flows from the large streamer head to the small patch, the potential drop transfers as well, and a short negative streamer starts to form and reaches a high electric field amplitude up to $325 \mathrm{kV} / \mathrm{cm}$ (see Movie S1 in the supporting information). As the short streamer develops, the radius increases causing the electric field to decrease, returning to the normal value of the previous long streamer. The maximum field value reached appears to be higher than the thermal runaway threshold $270 \mathrm{kV} / \mathrm{cm}$ as defined by the maximum friction
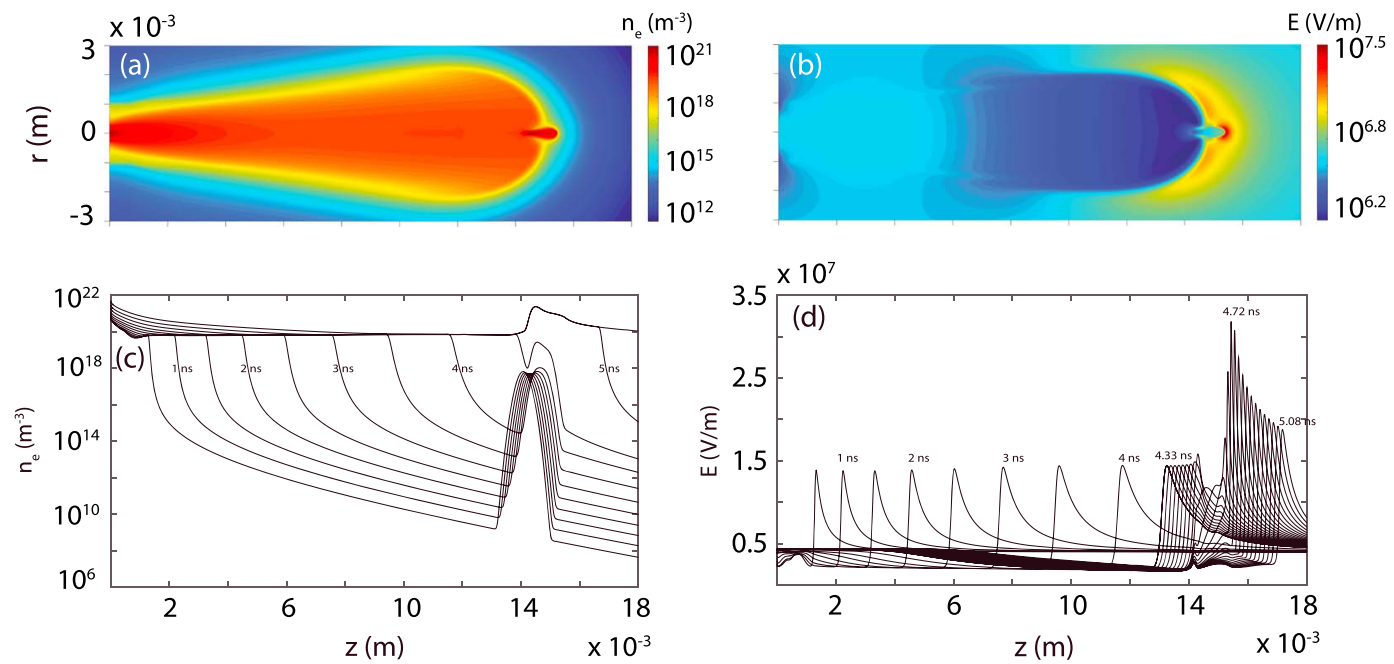

Figure 5. Cross-sectional view of the electron density (a) and electric field (b) at $4.72 \mathrm{~ns}$ in the case of $E_{0}=-40 \mathrm{kV} / \mathrm{cm}$, respectively. Profile of the electron density (c) and the electric field along the $z$ axis (d) in the case of $E_{0}=-40 \mathrm{kV} / \mathrm{cm}$, respectively. In panel (d) results are shown with a time step of 0.5 and $0.03 \mathrm{~ns}$ after $t=4.3 \mathrm{~ns}$. 


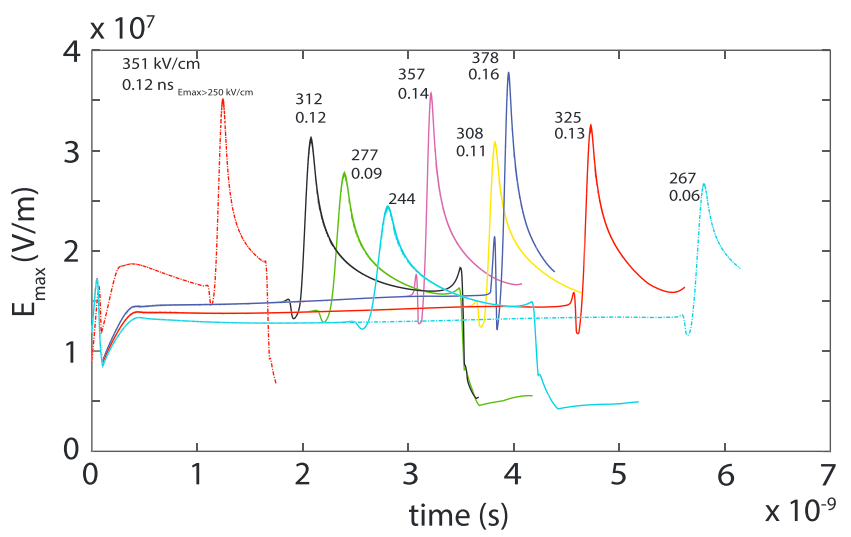

Figure 6. Evolution of the maximum electric field $E_{\max }$ as function of time. Black, violet, and blue curves correspond to $\left(E_{0}, d_{\text {patch }}\right)=(-45 \mathrm{kV} / \mathrm{cm}, 0.5$ $\mathrm{cm}) ;(-45,0.5) ;(-45,1.4)$, respectively. Green, yellow, and red curves correspond to $\left(E_{0}, d_{\text {patch }}\right)=(-40,0.5) ;(-40,1) ;(-40,1.4)$, respectively. Cyan and cyan dashed curves correspond to $\left(E_{0}, d_{\text {patch }}\right)=(-35,0.5) ;(-35$, $1.4)$, respectively. Red dashed curve corresponds to $\left(E_{0}, d_{\text {patch }}\right)=(-28,0.5)$.

force at $\sim 150 \mathrm{eV}$ (e.g., Babich \& Stankevich, 1973; Babich, 2003; Green \& Peterson, 1968; Moss et al., 2006; Peterson \& Green, 1968).

Figure 6 shows the evolution of the maximum electric field in the simulation domain versus time for a variety of ambient electric fields and patch locations. The maximum electric field value obtained is between 244 and $378 \mathrm{kV} / \mathrm{cm}$ and it corresponds to the cases $\left(E_{0}=-35 \mathrm{kV} / \mathrm{cm}\right.$, $\left.d_{\text {patch }}=0.5 \mathrm{~cm}\right)$ and $(-45 \mathrm{kV} / \mathrm{cm}, 1.4 \mathrm{~cm})$, respectively. We see clearly that the maximum electric field increases with increasing the distance of the plasma patch for a given ambient electric field. The explanation for this is that the maximum electric field in the patch is controlled by the amount of the available potential drop in the long streamer head. The longer the streamer channel the larger the quantity $\int_{0}^{l}\left(E_{0}-E_{c}\right) \mathrm{d} l$ to be transferred to the patch. A large amount of potential difference concentrated in a small volume creates a strong local electric field. This phenomena is equivalent to two conducting spheres of different radii $r_{1}>r_{2}$ of potentials $U_{1}>U_{2}$ connected with a wire and while at the same potential $U_{1}^{\prime}=U_{2}^{\prime}$ the electric field ratio of the two spheres $\frac{E_{2}}{E_{1}}=\frac{r_{1}}{r_{2}}$ shows that the field is higher at the surface of the sphere of small radius. This is a good approach if one considers a streamer-sphere shape model (e.g., Gallimberti et al., 1974). However, in case of an accurate streamer description the geometry is more complex and a geometrical factor related to the streamer head shape must be taken into account in the above ratio. For instance, if a streamer is described as a long conducting filament (e.g., D'yakonov \& Kachorovskii, 1989) a logarithm of the length to the radius of the channel $\ln \left(\frac{l}{r}\right)$ must be included in that ratio as follows: $\frac{E_{2}}{E_{1}}=\frac{r_{1}}{r_{2}} \frac{\ln \left(\frac{l_{1}}{r_{1}}\right)}{\ln \left(\frac{l_{2}}{r_{2}}\right)}$, where $\left(l_{1}, l_{2}\right)$ and $\left(r_{1}, r_{2}\right)$ are the lengths and radii of the long and short streamers, respectively.

In the case of an inhomogeneous ambient field shown by the blue curve in Figure 4, the red dashed curve in Figure 6 shows an increasing streamer head field at $t<0.5 \mathrm{~ns}$ due to the large-sphere field enhancement and then a decreasing field as the streamer travel into a low ambient field region. The strong inhomogeneous ambient field produced by a large-sphere electrode seems efficient in reaching higher maximum electric fields of $351 \mathrm{kV} / \mathrm{cm}$ with a patch being located just at $5 \mathrm{~mm}$ than some of the homogeneous field cases.

Table 2 shows the streamer-patch collision parameters, the maximum electric field, the duration of the maximum field $>250 \mathrm{kV} / \mathrm{cm}$, the potential drop $\int_{0}^{l}\left(E_{0}-E_{c}\right) \mathrm{d} l$, the average streamer speed, and the ratio of the peak field $E_{\max }$ to the streamer head field $E_{h}$. The reference value of $E_{h}$ is taken as the value of streamer head electric field just before the encounter with the plasma patch. For a given ambient field the results clearly show an increase of the peak field and its duration versus the increase of the patch distance, the potential

Table 2

The Peak Electric Field $E_{\max }$, the Duration of the Peak Electric Field $E_{\max }>250(\mathrm{kV} / \mathrm{cm}) t_{\max }$, the Potential Drop $\int_{0}^{l}\left(E_{0}-E_{c}\right) d l$, the Average Streamer Speed $V_{\text {str }}=\frac{l}{t}$, and the Ratio $\frac{E_{\max }}{E_{h}}$ for each Case in Figure 6

\begin{tabular}{|c|c|c|c|c|c|}
\hline $\begin{array}{l}E(\mathrm{kV} / \mathrm{cm}), \\
d_{\text {patch }}(\mathrm{cm})\end{array}$ & $\begin{array}{c}E_{\max } \\
(\mathrm{kV} / \mathrm{cm})\end{array}$ & $\begin{array}{l}t_{\max } \\
\text { (ns) }\end{array}$ & $\begin{array}{c}\int_{0}^{l}\left(E_{0}-E_{c}\right) \mathrm{d} l \\
(\mathrm{kV})\end{array}$ & $\begin{array}{c}V_{\text {str }} \times 10^{6} \\
(\mathrm{~m} / \mathrm{s})\end{array}$ & $\frac{E_{\max }}{E_{h}}$ \\
\hline$-35,0.5$ & 244 & 0 & 9 & 2 & 1.91 \\
\hline$-35,1.4$ & 267 & 0.06 & 15 & 2.5 & 2.01 \\
\hline$-40,0.5$ & 277 & 0.09 & 9.4 & 2.4 & 2.01 \\
\hline$-40,1$ & 308 & 0.11 & 14.7 & 2.8 & 2.15 \\
\hline$-40,1.4$ & 325 & 0.13 & 15.5 & 3 & 2.26 \\
\hline$-45,0.5$ & 312 & 0.12 & 9.7 & 2.8 & 2.11 \\
\hline$-45,1$ & 357 & 0.14 & 15.3 & 3.3 & 2.33 \\
\hline$-45,1.4$ & 378 & 0.16 & 16.2 & 3.7 & 2.44 \\
\hline$-28,0.5$ & 351 & 0.12 & 13 & 4.7 & 2.13 \\
\hline
\end{tabular}




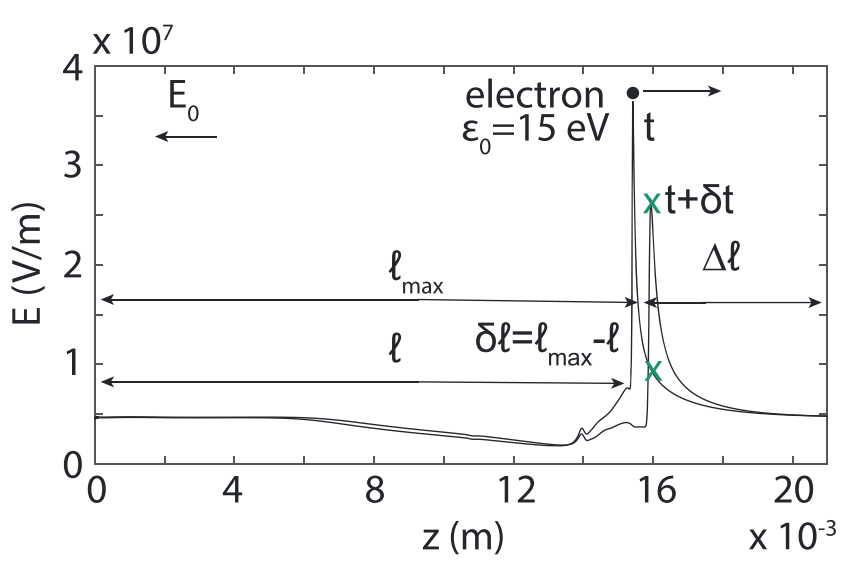

Figure 7. Illustration of the acceleration step of a test electron in a space-/time-varying streamer electric field. The results are taken from the case $(-45 \mathrm{kV} / \mathrm{cm}, 1.5 \mathrm{~cm})$ with a large time step $100 \mathrm{ps}$ to show enough spatial scale. The quantities $l_{\max }, l, \delta l, \Delta l, \mathrm{t}, \delta t$, and $E_{0}$ are the maximum streamer length when the maximum peak electric field is reached, the streamer reference length where the electric field suddenly increases, the streamer radius, the distance traveled by the runaway electron, the reference time for the streamer electric field data, the time step between two successive streamer electric field profiles, and the ambient electric field. The green cross shows two different values of the electric field at the same location when the dynamics of the streamer is taken into account. drop, and the streamer speed. Note that the quantity $\int_{0}^{l}\left(E_{0}-E_{c}\right) \mathrm{d} l$ is estimated just before the collision stage and the integration is made from the surface of the sphere electrode up to $l=l_{\max }-\delta R$ where $\delta R$ is by definition the streamer radius or the thickness of the streamer head charge layer.

\subsection{Estimation of the Energy and the Number of Thermal Runaway Electrons in a Streamer-Patch Collision}

To estimate the energy of thermal runaway electrons we solve numerically the deterministic equation (3) for a single test electron.

$$
m_{e} \frac{\mathrm{d} v_{e}\left(z_{e}, t\right)}{\mathrm{d} t}=q E\left(z_{e}, t\right)-F\left(\varepsilon\left(z_{e}, t\right)\right)
$$

where the quantities $m_{e}, v_{e}\left(z_{e}, t\right), E\left(z_{e}, t\right), F(\varepsilon)$, and $z_{e}$, are the electron mass, the electron velocity, the streamer electric field, the friction force, and the electron coordinate along the $z$ axis of the streamer, respectively.

This deterministic approach has been used by several authors to study runaway electrons (e.g., Babich \& Stankevich, 1973; Gurevich \& Zybin, 2001; Gurevich et al., 1992, 2007; Luque, 2017; Zubarev et al., 2017). This approach maximizes the energy of the electron as it does not take into account the angular scattering, and the electron is assumed to travel parallel to the central axis of the streamer electric field. The effect of the angular scattering, the diffusion, and the cross section has been studied in detail in the framework of runaway electrons (e.g., Chanrion et al., 2016, and references therein). The estimation is still reasonable as it is made over short distances of the order of $2-5 \mathrm{~mm}$ and in a nonrelativistic regime. The forward scattering for the electrons $>50 \mathrm{eV}$ prevail over the angular one (Pasko, 2006, pp. 261-265) especially in strong fields considered in this study (Babich et al., 1990, and references therein, p.523). Compared to the relativistic electrons traveling long distances the summation over all the small deviations is important and the angular scattering impacts the electron trajectory (Dwyer et al., 2012; Figure 7). As simulations are time consuming, we estimate just the lower and upper energy limits and we focus on the following cases $\left(E_{0}(\mathrm{kV} / \mathrm{cm}), d_{\text {patch }}(\mathrm{cm})\right)$ $=(-45,1.4),(-28,0.5)$, and $(-35,1.4)$.

The friction force $F(\varepsilon)$ that includes all the electron collision processes is tabulated as function of the electron energy based upon Moss et al. (2006). The maximum friction force is $260 \mathrm{keV} / \mathrm{cm}$ for an electron energy of $\sim 120 \mathrm{eV}$. We use a numerical time step of $\delta t_{\text {num }}=10^{-15} \mathrm{~s}$ to solve equation (3). A single test electron with an initial energy $\varepsilon_{0}=15 \mathrm{eV}$ is placed at the streamer tip (Figure 7) and then it is accelerated in the streamer electric field $E\left(z_{e}, t\right)$ every step $\delta t_{\text {num. }}$. At every step $\delta t_{\text {num }}$ the electric field $E\left(z_{e}, t\right)$ and the friction force $F(\varepsilon)$ are updated. To take into account the change of the electric field due to the moving streamer, we use streamer electric field data separated by a time step $\delta t$ of the order of a picosecond (ps) to capture accurate streamer electric field changes. When the electron travels $n_{\text {iter }} \delta t_{\text {num }}>\delta t$ where $n_{\text {iter }}$ is the number of numerical iterations, a streamer electric profile is update with a new one. The time steps are $0.2 \mathrm{ps}$ for the cases $(-35,1.4)$ and $(-28,0.5), 1 \mathrm{ps}$ for the cases $(-45,1.4)$ and $(-28,0.5)$. We choose 0.2 and 1 ps to run the fastest simulation $(-28,0.5)$ and to quantify the time step effect on the energy of the electron. Note that, if one uses the results of Figure 6 (the streamer head peak field) and assumes the electric field is distributed homogeneously following the approach of (Ihaddadene \& Celestin, 2015, section 3.3) the electron synchronizes with the peak electric field and the energy is going to be overestimated. The runaway electrons produced at the streamer head exit the head and they follow a decreasing electric field $\sim E\left(\frac{1}{r}\right)$.

Figure 8a shows the space and time-varying electric field traveled by a single test electron versus time. The electron acceleration is stopped when the electric field value falls off to $50 \mathrm{kV} / \mathrm{cm}$. The black curve corresponds to the $(-45,1.4)$ case with a 1-ps time step between the data files, and blue and red curves correspond to the $(-28,0.5)$ case with 0.2 - and 1-ps time steps, respectively. We first tested a single electron in a static streamer electric field and found that the electron failed to accelerate because of the lack of the available potential difference in the small radius of the short negative streamer as one can see from Figure $5 \mathrm{~d}$. However, once the streamer propagation is taken into account the electron is accelerated and gained energy. This 

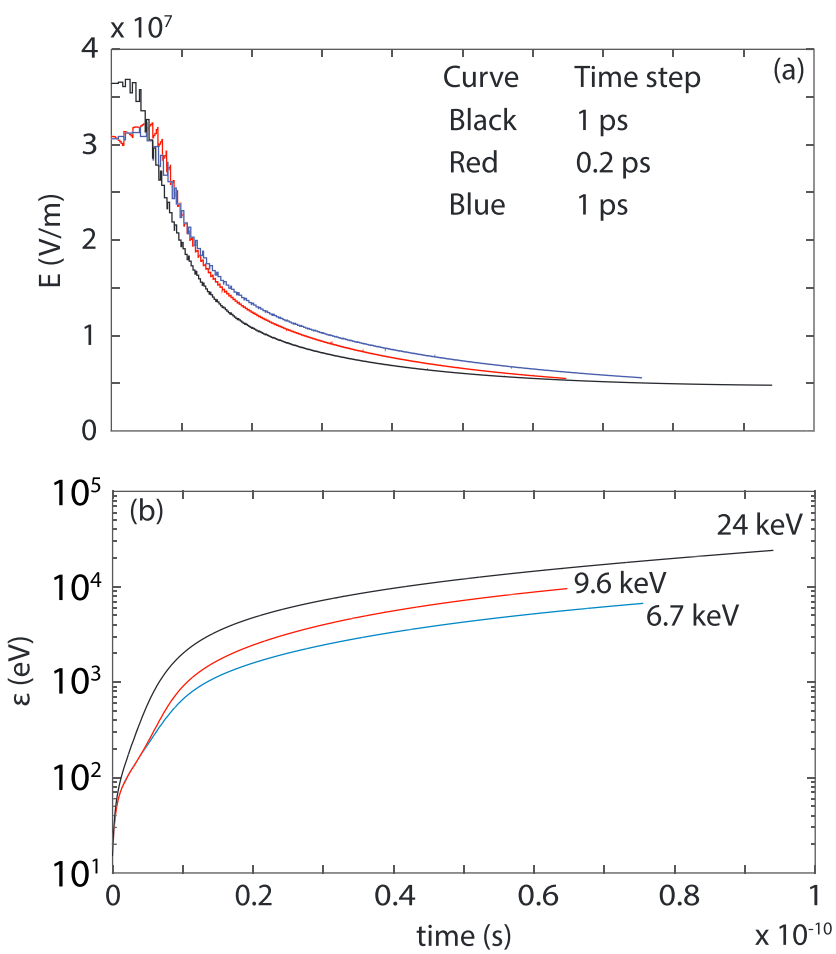

Figure 8. (a) Evolution of the space- and time-varying streamer electric traveled by a single test electron versus time. The black curve corresponds to the case $\left(E_{0}, d_{\text {patch }}\right)=(-45 \mathrm{kV} / \mathrm{cm}, 1.4 \mathrm{~cm})$, and the time step between two successive electric field data files is $1 \mathrm{ps}$. The blue and red curves correspond to $\left(E_{0}, d_{\text {patch }}\right)=(-28 \mathrm{kV} / \mathrm{cm}, 0.5 \mathrm{~cm})$, and time steps are 1 and 0.2 ps, respectively. (b) Evolution of the single test electron energy in the three cases versus time.

latter refers to a self acceleration mechanism introduced first by Babich (1982a, 1982b; Babich et al., 1990, p.534) and later on studied by Moss et al. (2006, section 4.2) who estimated a self-acceleration upper energy limit contribution no more than $9 \mathrm{keV}$ by Monte Carlo simulations. The electron can gain more energy than the available potential difference if that electron stays surfing in the streamer head for a sufficient time. A streamer speed of $\sim 10^{7} \mathrm{~m} / \mathrm{s}$ corresponds to an electron energy of $\sim 280 \mathrm{eV}$. Some of the electrons $>120$ up to $280 \mathrm{eV}$ amplify their energy gain while traveling within the high field region of the streamer head.

At the end of the electron acceleration we estimate $124 \mathrm{eV}, 6.7 \mathrm{keV}, 9.6 \mathrm{keV}$, and $24 \mathrm{keV}$ for the cases $(-35$, $1.4),(-28,0.5),(-28,0.5)$, and $(-45,1.4)$, respectively (Figure $8 \mathrm{~b})$. The values $124 \mathrm{eV}, 6.7 \mathrm{keV}, 9.6 \mathrm{keV}$, and $24 \mathrm{keV}$ were computed with $\delta t=0.2-, 1-, 0.2-$, and 1-ps data time steps, respectively. The $24-\mathrm{keV}$ electron reaches $200 \mathrm{keV}$ in an ambient field of $45-30 \mathrm{kV} / \mathrm{cm}$ over $4-7 \mathrm{~cm}$, respectively. We stop the electron acceleration when the electron reaches a location ahead of the streamer where the electric field is $\sim 50 \mathrm{kV} / \mathrm{cm}$. We do not show the results for the case $(-35,1.4)$ because the electron reaches $124 \mathrm{eV}$ and then loses its energy. If a very small time step is used the above energy increases by $43 \%$ up to $177 \mathrm{eV}$ as one can see from the

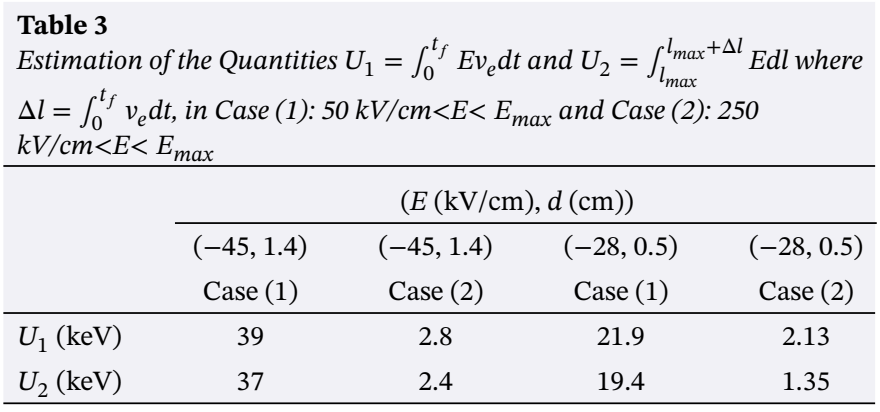


Table 4

The Number of Runaway Electrons $N_{\text {run }}$, the Flux of Runaway Electrons $\Phi$, and the Number of Streamer-Patch Collisions $N_{\text {coll }}$ Required to Explain the Experimental Results for each Case in Figure 6

\begin{tabular}{lcccccccc}
\hline$E(\mathrm{kV} / \mathrm{cm}), d_{\text {patch }}(\mathrm{cm})$ & $-35,0.5$ & $-35,1.4$ & $-40,0.5$ & $-40,1$ & $-40,1.4$ & $-45,0.5$ & $-45,1$ & $-45,1.4$ \\
\hline$N_{\text {run }} \times 10^{3}$ & 0 & 0.105 & 0.324 & 13 & 68 & 14 & 550 & 1870 \\
$\Phi=\frac{N_{\text {run }}}{t_{\max }}\left(\mathrm{s}^{-1}\right) \times 10^{12}$ & 0 & 1.75 & 3.6 & 181.2 & 523 & 116.7 & $3,437.5$ & $1,168.8$ \\
$N_{\text {coll }}=\frac{10^{6}}{N_{\text {run }}}$ & - & $10^{4}$ & $10^{3}$ & 77 & 15 & 71 & 280 \\
\hline
\end{tabular}

results of $(-28,0.5)$ case. The electron energy increased from 6.7 to $9.6 \mathrm{keV}$ when changing the time step from 1 to $0.2 \mathrm{ps}$.

We estimate the quantity $U_{1}=\int_{0}^{t_{f}} E v_{e} \mathrm{~d} t$, where $E$ and $v_{e}$ are the space- and time-varying streamer electric field and the electron velocity, respectively. The quantity $t_{f}$ stands for the end time of the electron acceleration and can be read from Figure 8. We compare $U_{1}$ with the quantity $U_{2}=\int_{l_{\max }}^{l_{\max }+\Delta l} E \mathrm{~d} l$ where $E$ is a single space-varying streamer electric field profile. The quantity $\Delta l=\int_{0}^{t_{f}} v_{e} \mathrm{~d} t$ is the distance traveled by an electron when it is accelerated. In the case $(-45,1.4)$, we find $U_{1}=39 \mathrm{kV}$ versus $U_{2}=7 \mathrm{kV}$, and $2.8 \mathrm{kV}$ versus $2.4 \mathrm{kV}$ if we estimate both quantities in the following electric field interval: $250 \mathrm{kV} / \mathrm{cm} \leq E \leq E_{h} \mathrm{kV} / \mathrm{cm}$. In the case $(-28,0.5)$ with a 1-ps time step we find $21.9 \mathrm{kV}$ versus $19.4 \mathrm{kV}$, and $2.13 \mathrm{kV}$ versus $1.35 \mathrm{kV}$. One sees differences between the quantities $U_{1}$ and $U_{2}$ of $2,2.5$, and $0.4 \mathrm{kV}, 0.78 \mathrm{kV}$ when $250 \leq E \leq E_{h} \mathrm{kV} / \mathrm{cm}$ for the cases $(45,1.4)$ and $(28,0.5)$, respectively. The values of $U_{1}$ and $U_{2}$ are also summarized in Table 3. From the estimations above, one can find an average energy loss over the $\sim 5.5-\mathrm{mm}$ distance traveled by the electron of $\varepsilon_{\text {loss }}=\frac{(39 \mathrm{keV}-24 \mathrm{keV}}{5.5 \mathrm{~mm}} \simeq 2.7 \mathrm{keV} / \mathrm{mm}$ and so $\sim 190 \mathrm{eV}$ per streamer radius $\delta l \sim 7 \times 10^{-5} \mathrm{~m}$. The average energy gain is $\varepsilon_{\text {gain }}=\frac{24}{5.5} \simeq 4 \mathrm{keV} / \mathrm{mm}$ and it corresponds to $280 \mathrm{eV}$ per streamer radius. It appears that the energy difference of $400 \mathrm{eV}$ up to $800 \mathrm{eV}$ (in the case $E_{h} \geq 250 \mathrm{kV} / \mathrm{cm}$ ) supplied by the streamer dynamics is a helpful boost for the low-energy electrons $\sim 120 \mathrm{eV}$ to escape the thermal zone to the side of the runaway one. Figure 7 clearly shows how tiny the streamer radius $\delta l$ is and that the electric field at the same location marked by a green cross can drastically jump from 100 up to $250 \mathrm{kV} / \mathrm{cm}$ while the streamer dynamics is taken into account.

The number of thermal runaway electrons is estimated based on the equation (4) (e.g., Babich et al., 2015; Bakhov et al., 2000).

$$
N_{\text {run }}=\int_{0}^{t_{\max }} \int_{V} v_{\text {run }} n_{\mathrm{e}} \mathrm{d} V \mathrm{~d} t
$$

where $v_{\text {run }}(E)=3.5 \times 10^{-24} \exp \left(-\left(2.166 \times 10^{-7} \times \mathrm{E}\right)^{2}+3.77 \times 10^{-6} \times \mathrm{E}\right)$ is the runaway electron frequency calculated by a Monte Carlo simulation (Bakhov et al., 2000).

The integration is made over a field region $E \geq 250 \mathrm{kV} / \mathrm{cm}$ and over an interval of time $t_{\max }$ (see Table 2). The time step between data files used to make this estimation is $0.01 \mathrm{~ns}$. Table 4 lists the estimated number of runaway electrons, their average flux, and the number of streamer-patch collisions required to explain the experimental results of Kochkin, Köhn, et al. (2016) for every case considered in the simulation. From the results we estimate an upper limit of the runaway electrons self-current and electric field created at the streamer front. We focus on the case $(-45,1.4)$ and we find a self-current $I=\frac{e N_{\text {run }}}{t_{\max }} \simeq 0.002 \mathrm{~A}$ and an average self-electric field $E=\frac{e N_{\mathrm{run}} r_{\mathrm{str}}}{\left(\frac{4}{3} \pi r_{\mathrm{str}}^{3}\right) 3 \epsilon_{0}}=\frac{e N_{\mathrm{run}}}{4 \epsilon_{0} S_{\mathrm{str}}} \simeq 6 \mathrm{kV} / \mathrm{cm}$ assuming a spherical geometry. A runaway electron at the front of the streamer creates low-energy electrons along its trajectory. This additional ionization due to runaway electrons combined self consistently with the ionization due to low-energy thermal electrons may increase the speed of the ionization front. The increase of streamer speed by the runaway electrons was already suggested and discussed by Aleksandrov et al. (1995, p.72, and references therein).

\section{Discussion}

Since the suggestion of the streamer head-on collision as the process behind the production of thermal runaway electrons and X-ray emissions by laboratory spark discharges and by lightning stepped leaders (e.g., Cooray et al., 2009; Kochkin et al., 2012), it became a hot topic within the community of atmospheric and space electricity. It may also be a source of VHF-UHF emissions from lightning (e.g., Montanyà et al., 2015; 
Luque, 2017; Shi et al., 2018, 2019). Recently, Babich and Bochkov (2017) made an interesting analysis of Ihaddadene and Celestin (2015) results from the point of view of a possible underestimation effect of the calculated electric field by the applied conducting boundary conditions as suggested by Kochkin, Lehtinen, et al. (2016) and by the used transport coefficients data from Morrow and Lowke (1997). Babich and Bochkov (2017) came up with no effect on the conclusion that conventional streamer head-on collision is unlikely a source of runaway electrons. Kochkin, Lehtinen, et al. (2016), Lehtinen, Kochkin, and Ostgaard (2016), and Lehtinen and Ostgaard (2016) used point-to-point and point-to-plane electrodes discharge 2-D and 1-D models and came to a similar conclusion. Several modeling attempts of a single head-on collision have been conducted (e.g., Babich \& Bochkov, 2017; Ihaddadene \& Celestin, 2015; Köhn et al., 2017; Lehtinen, Kochkin \& Ostgaard 2016; Lehtinen \& Ostgaard 2016; Luque, 2017) with a variety of modeling complexities and it appears that the general idea does not seem to be explaining the experimental observations.

The maximum electric field in a head-on collision can be approximated as $E_{\max } \simeq \frac{\left(\int_{0}^{l^{-}}\left(E_{0}-E_{c}^{-}\right) \mathrm{d} l+\int_{0}^{l^{+}}\left(E_{0}-E_{c}^{+}\right) \mathrm{d} l\right)}{(\delta R)}$, with $\delta R=\delta R^{+}+\delta R^{-}, l$, and $\delta R$ are the size of the high field region, the streamer length, and the streamer radius, respectively. The plus and minus signs stand for positive and negative streamers. As the streamers approach each other they squeeze the available potential drop into a short region $\delta R$ and the electric field is increased. However, the field increase has a physical limit because of the local conductivity and the size of the high field zone. When the Maxwell time $\tau_{m}$ becomes smaller than the ionization time $\tau_{i}$, then the field starts to collapse (Celestin \& Pasko, 2011, see Figure 7). If one assumes $\tau_{i}=\frac{1}{v_{i}}$ and $\tau_{m} \sim \frac{\varepsilon_{0}}{q \mu_{e} n_{e_{0}}} \exp \left(-v_{i} t\right)$, one sees that the Maxwell time decreases faster than the ionization time at a certain level of the electron density $n_{e_{0}}$ in between the streamer heads. The photoionization rate also affects the ionization time as long as the distance between the two streamer heads is still larger than the photoionization characteristic length. The field increase is streamer speed dependent, and the faster the collision the faster the field increases and vice versa. At extremely high field values such as $400 \mathrm{kV} / \mathrm{cm}$, to produce a $100-\mathrm{keV}$ electron, the average characteristic distance required is $4 \mathrm{~cm}$, which is higher than a realistic maximum high field zone of $0.4 \mathrm{~cm}$ formed by two large streamers at their branching stage (e.g., Celestin \& Pasko, 2011). Even with large colliding streamers, $100-\mathrm{keV}$ electrons are unlikely to be produced. Moreover, the $\sim 1-\mathrm{keV}$ electron energy found by Monte Carlo and PIC simulations of streamer head-on collisions (e.g., Ihaddadene \& Celestin, 2015; Köhn et al., 2017) requires a channel electric field $E_{c}=100 \mathrm{kV} / \mathrm{cm}$ to run away. The runaway electron moves against the approaching high field front and their residence time in the high field zone is always shorter than the maximum field duration $t_{\max }$.

The multistreamer head-on collisions problem is beyond the single-streamer head-on collision complexity. It is a combination of high number of streamers propagating in an electromagnetic, kinetic, and hydrodynamic environment that we are not able to simulate with the current available models if simplifications are not made. That is the reason why in the present work we use a simplified multiple ellipsoids approach to test how likely the head-on collision is a source of runaway electrons. Indeed, the dynamics of the electric field in a meter-scale discharge is very complex, and our model does not capture the details of streamer collective effects. However, from the present results we find two issues that prevent the electrons from running away when short and large dielectric ellipsoids representative of streamers are used. The short ellipsoids possess a limited potential drop and electrons do not gain enough energy to run away, and the long ellipsoids possess a large potential drop but then electrons fall into the long channel's low electric field and they lose the energy they gained at the collision point. Based on Table 1, we would require a single head-on collision to produce electrons $\varepsilon_{\text {run }}>60 \mathrm{keV}$ at the collision point to allow them to runaway in $E_{c}=10 \mathrm{kV} / \mathrm{cm}$ positive streamer channel, if one assumes an average discharge ambient field of $\simeq 20 \mathrm{kV} / \mathrm{cm}$. From Table 1 , one sees that the low electric field requires a high-energy $\varepsilon_{\text {run }}$ for an electron to run away. A self-consistent particle model taking into account the ionization from runaway electrons (Köhn et al., 2017, see Figure 4) found one electron with $\varepsilon \sim 500 \mathrm{eV}$ that is unlikely to runaway in $\sim 20 \mathrm{kV} / \mathrm{cm}$ positive streamer channel's field, which requires $\varepsilon_{\text {run }}>40 \mathrm{keV}$. Unlike a short gap simulation of Köhn et al. (2017), Luque (2017) conducted a long plasma fluid streamer simulation over $\sim 30-\mathrm{cm}$ gap to achieve a large potential drop, and their one-dimensional deterministic acceleration produced an electron with $\varepsilon>5 \mathrm{keV}$ that is also not going to runaway in $E_{c} \sim 12 \mathrm{kV} / \mathrm{cm}$, which requires $\varepsilon_{\text {run }}>60 \mathrm{keV}$. We emphasize that there is not only the parameter of the available potential drop to take into account at the head-on collision location for the production of runaway electrons, but others, such as the size of the high field zone, the duration of the peak field, and the energy of the runaway electrons, when they come out from the collision zone to flow through the channel, 

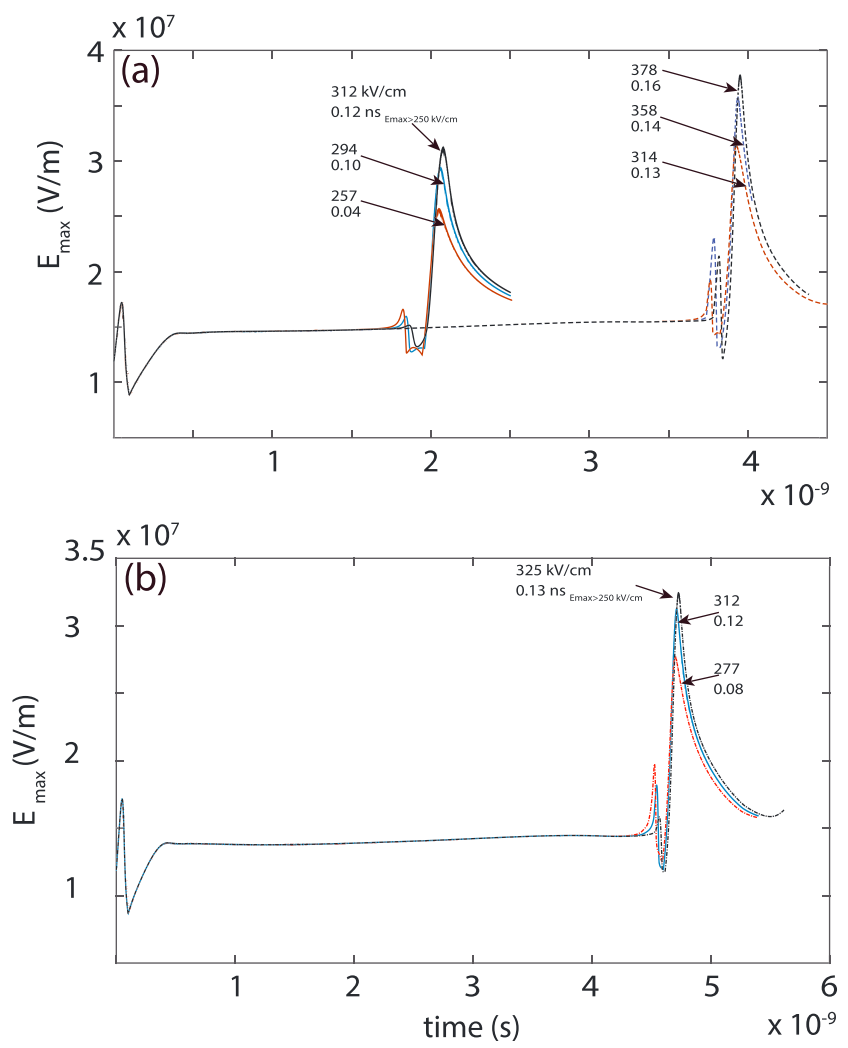

Figure 9. (a) Evolution of the maximum electric field $E_{\max }$ as function of time. Black, dashed black, blue, dashed blue, and red, dashed red, curves correspond to patch sizes $\sigma_{r}(\mu \mathrm{m})=15,40,80$, and $E_{0}, d_{\text {patch }}=-45 \mathrm{kV} / \mathrm{cm}$, $0.5 \mathrm{~cm} ;-45,1.4$, respectively. (b) Evolution of the maximum electric field $E_{\max }$ as function of time. Dashed black, blue, and dashed red, curves correspond to patch sizes $\sigma_{r}(\mu \mathrm{m})=15,40,80$, and $E_{0}, d_{\text {patch }}=-40 \mathrm{kV} / \mathrm{cm}$, $1.4 \mathrm{~cm}$, respectively. are also important. Streamer head-on collisions are believed to happen in the middle of the discharge gap during the encounter of long negative and positive streamers (e.g., Kochkin et al., 2012). However, there is no strong experimental evidence for streamer collisions to occur and to produce X-rays (e.g., da Silva et al., 2017). The detector response time is much longer than the picosecond interaction times of a single-streamer head-on collision, so the detector cannot pinpoint the source. At the present time only a statistical correlation between the X-ray emissions and the encounter of long streamers has been reported as a possible evidence (e.g., Kochkin et al., 2012).

Figure 1 shows a complex morphology of the streamer zone near the point electrode when X-ray emissions are produced. The figure clearly shows different streamer channel sizes, and small aligned isolated dots probably formed after the decay of the streamer channel. Some of the dots act as inhomogeneities initiating upward propagating positive streamers. From the results presented in this work, we believe that a collision between a large negative streamer and a plasma patch similar to those in Figure 1 is a better candidate for explaining the production of runaway electrons and the associated $\mathrm{X}$-ray emissions. The plasma patches used in this study have a size $\sigma_{z}=200$ and $\sigma_{r}=15 \mu \mathrm{m}$. We may ask the question why the patch is so thin radially and also whether a numerical resolution of $8 \mu \mathrm{m}$ could resolve accurately such a thin patch. We have run a numerical test with $\sigma_{r} \sim 5 \times \Delta_{r}\left(\Delta_{r}=3 \mu \mathrm{m}\right)$, and the results do not show a change that would affect the principal conclusions of this work. The maximum field is increased by $1 \%$ at $3-\mu \mathrm{m}$ resolution with respect to the $8-\mu \mathrm{m}$ resolution case. In addition to that, we have also conducted additional simulations with different patch sizes $\sigma_{r}=40$ and $80 \mu \mathrm{m}$ (same $\sigma_{z}=200 \mu \mathrm{m}$ ) at locations $z=0.5$ and $1.4 \mathrm{~cm}$ in -45 and $40 \mathrm{kV} / \mathrm{cm}$ ambient electric fields (sphere-to-plane electrode configuration), and the results are shown in Figure 9. The results clearly show strong electric fields similar to the values obtained using a patch size $\sigma_{r}=15 \mu \mathrm{m}$ that are sufficient to generate thermal runaway electrons. One sees that the maximum electric field is decreased by $\sim 15-20 \%$ when increasing the patch radial size from 15 to $80 \mu \mathrm{m}$. The origin and the upper and lower size limits of the patch are both uncertain. We believe that the patch might be produced either by a decay of former streamer channels created when streamers propagate into a low ambient field volume (e.g., $\sim 95 \mu \mathrm{m}$ radius for a thinnest streamer (Briels et al., 2006)) or by a development of a group of polarized electron avalanches/pilots that act as initiation points for positive and negative streamers (e.g., Reess et al., 1995, section 3). As far as we know, the characteristic size that corresponds to an avalanche to streamer transition for atmospheric air is about 30-50 $\mu \mathrm{m} / 2$ to $15-25 \mu \mathrm{m}$ (e.g., Naidis, 1996; Qin, 2013, p.28-31).

We choose a sharper ellipsoidal shape for the plasma patch because it produces a stronger peak electric field than a spherical shape. The analysis by Østgaard et al. (2016) showed that runaway electrons are likely to be produced at tens of centimeters near the electrode zone where the electric field is believed to be $\geq E_{k}$. From the Figure 2 of Kochkin et al. (2012) one can estimate a $50 \mathrm{kV} / \mathrm{cm}$ to span over $16 \mathrm{~cm}$ at $\sim 0.8 \mathrm{MV}$ applied voltage when X-rays are produced. Those studies support our selection for the ambient electric fields used in the present paper. We conducted simulations in a low ambient electric field $\sim 20-25 \mathrm{kV} / \mathrm{cm}$, in a $\sim 2-\mathrm{cm}$ gap, and we obtained with a $\sim 12-\mathrm{kV}$ potential drop a peak electric field up to $\sim 180 \mathrm{kV} / \mathrm{cm}$, which is below the $250 \mathrm{kV} / \mathrm{cm}$ required for the production of thermal runaway electrons. The low ambient field cases $\left(<E_{k}\right)$ require longer streamers to achieve larger potential drops and higher runaway threshold energy $(\varepsilon>40 \mathrm{keV}$, see Table 1). For instance, a potential drop $U=40-100 \mathrm{kV}$ requires a streamer length $l \simeq \frac{U}{\left(E_{0}-E_{c}\right)} \simeq 5-10 \mathrm{~cm}$ that is beyond our simulation capabilities. Furthermore, according to Celestine and Pasko (2011, section 2) when the field in the streamer channel approaches the stability field $(10-15 \mathrm{kV} / \mathrm{cm})$ in the case of a negative streamer, the streamer head moves with a constant potential drop and does not expand. In addition, the streamer speed is lower in ambient fields $<E_{k}$, which results in a slower and softer collision with a weaker 
peak electric field. In low ambient electric fields a long streamer develops a large radius to host a large potential drop and keep a stable head electric field as it propagates (see properties of stable streamers; e.g., Qin \& Pasko, 2014). As the electric field at the front of the streamer head follows $\sim \frac{1}{r_{s}}$ profile, the plasma patch is expected to polarize and initiate streamers in a quasi-homogeneous streamer electric field before the full collision occurs, which will prevent the short streamer from reaching high peak electric fields. The streamer residence time in the plasma patch $\frac{l_{\text {patch }}}{V_{\text {str }}}$ must be short enough to develop a fast ionization growth to increase the peak electric field in the patch. The thermal runaway electrons are easy to produce in this fashion in ambient electric fields $\geq E_{k}$ where streamers expand and carry large potential differences. The low ambient field case deserves to be explored in future studies.

Based on the results of Kochkin et al. (2014) X-rays are not produced at the first burst of the streamer but at the fourth burst. This may indicate that a specific set of conditions needs to be filled. The first burst of streamers propagates short distances with low speed and dies off (e.g., Morrow \& Lowke, 1997; Qin \& Pasko, 2014) as the ambient field far away from the electrode approaches $\sim 0 \mathrm{kV} / \mathrm{cm}$. The first burst of streamers creates a preionized medium for the next coming bursts. Both voltage and current increase continuously while the previous streamer channels decay over the attachment time scale. If one assumes a streamer channel electron density $10^{20} \mathrm{~m}^{-3}$, a streamer channel field of $25 \mathrm{kV} / \mathrm{cm}$, and an ambient field $50 \mathrm{kV} / \mathrm{cm}$, it takes $50 \mathrm{~ns}$ for a streamer to travel $\sim 15 \mathrm{~cm}\left(3 \times 10^{6} \mathrm{~m} / \mathrm{s}\right)$ and for the density to go down to $10^{18} \mathrm{~m}^{-3}$ and to form plasma inhomogeneities. Within the decay process the field rises again with the voltage, and if a collision occurs, it is going to be the adequate moment for the X-ray emissions to be produced. We do not expect the X-ray emissions to be produced within the first burst of streamers because the plasma patches do not exist yet. The frequency of streamer-patch collisions determines the number of both the runaway electrons and the associated X-ray photons. From Table 4, one collision in a case $(-45 \mathrm{kV} / \mathrm{cm}, 1.4 \mathrm{~cm})$ is sufficient to produce the $10^{6}$ runaway electrons with $\sim 100 \mathrm{keV}$ per one electron to explain the $10^{4} \mathrm{X}$-ray photons based on 100 electrons per a single X-ray photon (Kochkin, Köhn, et al., 2016). The lower ambient field demands a higher number of collisions. Tens of collisions is a reasonable number to consider. However, it is hard to imagine a higher number because of the many parameters that control the streamer-patch mechanism to occur such as the streamer length, the speed, the homogeneous or inhomogeneous ambient field, the streamers density per burst, the characteristic decay time of the streamer channel, and the shape of the plasma patch, make it unlikely for all the collisions to produce runaway electrons and the associated X-rays.

The estimation of the energy and the number of thermal runaway electrons used in this work is an acceptable approximation to quantify the two quantities and computationally not expensive. We realize that the estimation is sensitive to the initial location of the test electron and the local high electric fields. We position the electron in the back $(E>250 \mathrm{kV} / \mathrm{cm})$ of the peak streamer field $(378 \mathrm{kV} / \mathrm{cm})$ in the case $(-45 \mathrm{kV} / \mathrm{cm}$, $1.4 \mathrm{~cm}$ ), and we find that the electron can gain without the streamer dynamics up to $20 \mathrm{keV}$. We do not expect a drastic change in the energy of the electron using a Monte Carlo approach as we already explained in section 3.3. The energies considered in the current study are at $4 \%$ maximum of the relativistic electron mass energy $(\sim 500 \mathrm{keV})$. From Table 1 , the Monte Carlo simulation requires $\varepsilon_{\text {run }}=15 \mathrm{keV}$ in $E_{0}=50 \mathrm{kV} / \mathrm{cm}$ and if one applies up to $30 \%$ less energy gain to the estimated value $\varepsilon=24 \mathrm{keV}$ due to Monte Carlo considerations one finds $\sim 17 \mathrm{keV}>\varepsilon_{\text {run }}=15 \mathrm{keV}$. The Monte Carlo model would give a better distribution of electrons for a broad range of energies as it has the ability to deal with a high number of electrons instead of a one single test electron method. However, the separate treatment of runaway electrons and streamer simulations makes the estimation not self consistent as it is in a particle code (e.g., Chanrion \& Neubert, 2010; Köhn et al., 2017). In case of high fields $>250-300^{\sim} \mathrm{kV} / \mathrm{cm}$ the drift-diffusion local field approximation is no longer valid and the relation $v_{e}(E)=\mu_{e}(E) E$ is violated as electrons are no longer in equilibrium in the local electric field; hence, the electron transport coefficient and the electron distribution are not well resolved. In addition, the amount of ionization due to the runaway electrons affects the streamer local electric field (Köhn et al., 2017, see Figure 2), the collision speed, the duration of the peak field, and the rate of runaway electrons with the associated X-ray photons. Li et al. $(2007,2008)$ set a limit between the self consistent particle and fluid models at $200 \mathrm{kV} / \mathrm{cm}$. At that field value the particle code describes better the streamer ionization front with a $60 \%$ higher ionization rate than the fluid models due to electrons $>15$ and $<120 \mathrm{eV}$ that are not runaway and create more ionization. The nonlocal approximation of Naidis (1997) may be an alternative solution in between the local approximation and particle approach to describe the electron distribution in this case. We also point that PIC techniques such as resampling or superparticle treatment of 
high number of electrons beyond the computation capabilities produce numerical enhanced ionization and branching effects (Chanrion \& Neubert, 2010, paragraph 36).

How the runaway electrons affect the streamer properties at high-energy regimes is still not fully understood. The runaway electrons may create electron avalanches at the front of the negative streamer head (e.g., Chanrion \& Neubert, 2008; Köhn, Chanrion, Babich, et al., 2018) and affect its velocity. Advanced simulations are required to investigate these effects and understand the mechanisms of the runaway electrons and $\mathrm{X}$-rays in laboratory discharges and lightning leaders. The ellipsoid model used in this study may not be an accurate description of the complex geometry of the electric field produced by a large number of streamers. The estimation of the energy and the number of runaway electrons produced by the streamer-patch mechanism is one dimensional and not self-consistent. A PIC simulation of multiple-streamer $(>2)$ head-on collisions to test the possibility of a continuous accelerations of thermal runaway electrons by other neighboring streamers and/or a reproduction of one of the streamer-patch configurations would be an interesting future work to do and compare with the results of this paper.

\section{Conclusions}

The main conclusions of this work can be summarized as follows:

1. We have combined dielectric ellipsoids with Monte Carlo simulations to study the conventional multiple-streamer head-on collisions and we have found that the runaway electrons lose their total energy in the positive streamer channel's low electric field. The positive streamer channel is an additional factor of energy loss for the electrons to overcome if they are produced by a streamer head-on collision.

2. We have simulated a collision between a large negative streamer and a small neutral plasma patch, and we have shown it to produce thermal runaway electrons in ambient electric fields $>E_{k}$.

3. We have found a number of runaway electrons in the streamer-patch simulations between $10^{2}$ and $10^{6}$ in ambient electric fields $\left|E_{0}\right|=35$ and $45 \mathrm{kV} / \mathrm{cm}$, with an average energy per electron up to $24 \mathrm{keV}$.

4. We have found that the streamer self-acceleration contributes to a runaway acceleration process.

\section{Acknowledgments}

This material is based upon work supported by the Air Force Office of Scientific Research under Award FA9550-16-1-0396. Sebastien Celestin's research is supported by the French space agency CNES in the framework of the satellite mission TARANIS. This work complies with the AGU data policy. All data used in this paper are available at https://zenodo.org/DOI: 10.5281/zenodo.2362416/zenodo. 2565279/zenodo.3239526. The authors thank L. P. Babich and the anonymous reviewers for their comments that improved this article.

\section{References}

Aleksandrov, G. N. (1966). Mechanism of corona-to-spark transition in long air gaps. Soviet Physics - Technical Physics, $10,948$.

Aleksandrov, N. L., Bazelyan, A. E., Bazelyan, E. M., \& Kochetov, I. V. (1995). Modeling of long streamers in atmospheric-pressure air. Plasma Physics Reports, 21, 57-75.

Arrayás, M., Ebert, U., \& Hundsdorfer, W. (2002). Spontaneous branching of anode-directed streamers between planar electrodes. Physical Review Letters, 88(17), 174502. https://doi.org/10.1103/PhysRevLett.88.174502

Babaeva, N. Y., \& Naidis, G. V. (1996a). Simulation of positive streamers in air in weak uniform electric fields. Physics Letters A, 215, 187-190. https://doi.org/10.1016/0375-9601(96)00225-3

Babaeva, N. Y., \& Naidis, G. V. (1996b). Two-dimensional modelling of positive streamer dynamics in non-uniform electric fields in air. Journal of Physics D: Applied Physics, 29, 2423-2431. https://doi.org/10.1088/0022-3727/29/9/029

Babich, L. P. (1982a). A new type of ionization wave and the mechanism of polarizational self-acceleration of electrons in gas discharges at high overvoltages. Doklady Akademii Nauk SSSR, 263, 76-79.

Babich, L. P. (1982b). Polarization selfacceleration of electrons. Soviet Journal of Plasma Physics, 8, 404-441.

Babich, L. P. (2003). High-energy phenomena in electric discharges in dense gases: Theory experiment and natural phenomena. Arlington, Virginia, USA: Futurepast Inc, 2003. Futurepast.

Babich, L. P. (2005). Analysis of a new electron-runaway mechanism and record-high runaway-electron currents achieved in dense-gas discharges. Physics-Uspekhi, 48, 1015-1037. https://doi.org/10.1070/PU2005v048n10ABEH002805

Babich, L., \& Bochkov, E. (2017). Numerical simulation of electric field enhancement at the contact of positive and negative streamers in relation to the problem of runaway electron generation in lightning and in long laboratory sparks. Journal of Physics D: Applied Physics, 50, 455202. https://doi.org/10.1088/1361-6463/aa88fd

Babich, L. P., Bochkov, E. I., Kutsyk, I. M., Neubert, T., \& Chanrion, O. (2015). A model for electric field enhancement in lightning leader tips to levels allowing X-and gamma ray emissions. Journal of Geophysical Research: Space Physics, 120, 5087-5100. https://doi.org/10. 1002/2014JA020923

Babich, L. P., Kutsyk, I. M., Donsko, E. N., \& Dwyer, J. R. (2013). Analysis of the experiment on registration of X-rays from the stepped leader of a cloud-to-ground lightning discharge. Journal of Geophysical Research: Space Physics, 118, 2573-2582. https://doi.org/10.1002/ jgra.50236

Babich, L. P., Loǐko, T. V., \& Tsukerman, V. A. (1990). Reviews of topical problems: High-voltage nanosecond discharge in a dense gas at a high overvoltage with runaway electrons. Soviet Physics Uspekhi, 33, 521-540. https://doi.org/10.1070/PU1990v033n07ABEH002606

Babich, L. P., \& Stankevich, Y. L. (1973). Transition from streamers to continuous electron acceleration. Soviet Physics - Technical Physics, $17,1333$.

Bakhov, K. I., Babich, L. P., \& Kutsyk, I. M. (2000). Temporal characteristics of runaway electrons in electron-neutral collision-dominated plasma of dense gases. Monte Carlo calculations. IEEE Transactions on Plasma Science, 28, 1254-1262. https://doi.org/10.1109/27.893314 Bazelyan, E. M., \& Raizer, Y. P. (1998). Park Discharge (pp. 291). Berlin Heidelberg New York: CRC.

Borukhov, M. Y., Bek-Bulatov, I. K., Lukashevich, L. L., Nagaibekov, R. B., \& Umurzakov, N. (1973). Modelling of field-amplification processes on cathode protuberances in vaccum arcs. Soviet Physics - Technical Physics, 17, 1199. 
Briels, T. M. P., Kos, J., van Veldhuizen, E. M., \& Ebert, U. (2006). Circuit dependence of the diameter of pulsed positive streamers in air. Journal of Physics D: Applied Physics, 39, 5201-5210. https://doi.org/10.1088/0022-3727/39/24/016

Bugaev, S. P., Litvinov, E. A., Mesyats, G. A., \& Proskurovskii, D. I. (1975). Reviews of topical problems: Explosive emission of electrons. Soviet Physics Uspekhi, 18, 51-61. https://doi.org/10.1070/PU1975v018n01ABEH004693

Celestin, S., \& Pasko, V. P. (2011). Energy and fluxes of thermal runaway electrons produced by exponential growth of streamers during the stepping of lightning leaders and in transient luminous events. Journal of Geophysical Research, 116, A03315. https://doi.org/10.1029/ 2010JA016260

Chanrion, O., Bonaventura, Z., Bourdon, A., \& Neubert, T. (2016). Influence of the angular scattering of electrons on the runaway threshold in air. Plasma Physics and Controlled Fusion, 58(4), 044001. https://doi.org/10.1088/0741-3335/58/4/044001

Chanrion, O., \& Neubert, T. (2008). A PIC-MCC code for simulation of streamer propagation in air. Journal of Computational Physics, 227, 7222-7245. https://doi.org/10.1016/j.jcp.2008.04.016

Chanrion, O., \& Neubert, T. (2010). Production of runaway electrons by negative streamer discharges. Journal of Geophysical Research, 115 A00E32. https://doi.org/10.1029/2009JA014774

Cooray, V., Arevalo, L., Rahman, M., Dwyer, J., \& Rassoul, H. (2009). On the possible origin of X-rays in long laboratory sparks. Journal of Atmospheric and Solar - Terrestrial Physics, 71, 1890-1898. https://doi.org/10.1016/j.jastp.2009.07.010

da Silva, C. L., Millan, R. M., McGaw, D. G., Yu, C. T., Putter, A. S., LaBelle, J., \& Dwyer, J. (2017). Laboratory measurements of X-ray emissions from centimeter-long streamer corona discharges. Geophysical Research Letters, 44, 11,174-11,183. https://doi.org/10.1002/ 2017GL075262

D'yakonov, M. I., \& Kachorovskii, V. Y. (1989). Streamer discharge in a homogeneous field. Zhurnal Eksperimental'noi i Teoreticheskoi Fiziki, 95, 1850-1859.

Dwyer, J. R. (2007). Relativistic breakdown in planetary atmospheres. Physics of Plasmas, 14(4), 042,901-042,901. https://doi.org/10.1063/ 1.2709652

Dwyer, J. R. (2012). The relativistic feedback discharge model of terrestrial gamma ray flashes. Journal of Geophysical Research, 117, A02308. https://doi.org/10.1029/2011JA017160

Dwyer, J. R., Rassoul, H. K., Al-Dayeh, M., Caraway, L., Chrest, A., Wright, B., et al. (2005). X-ray bursts associated with leader steps in cloud-to-ground lightning. Geophysical Research Letters, 32, L01803. https://doi.org/10.1029/2004GL021782

Dwyer, J. R., Rassoul, H. K., Saleh, Z., Uman, M. A., Jerauld, J., \& Plumer, J. A. (2005). X-ray bursts produced by laboratory sparks in air. Geophysical Research Letters, 32, L20809. https://doi.org/10.1029/2005GL024027

Dwyer, J. R., Schaal, M., Rassoul, H. K., Uman, M. A., Jordan, D. M., \& Hill, D. (2011). High-speed X-ray images of triggered lightning dart leaders. Journal of Geophysical Research, 116, D20208. https://doi.org/10.1029/2011JD015973

Dwyer, J. R., Smith, D. M., \& Cummer, S. A. (2012). High-energy atmospheric physics: Terrestrial gamma-ray flashes and related phenomena. Space Science Reviews, 173, 133-196. https://doi.org/10.1007/s11214-012-9894-0

Dwyer, J. R., Uman, M. A., Rassoul, H. K., Al-Dayeh, M., Caraway, L., Jerauld, J., et al. (2003). Energetic radiation produced during rocket-triggered lightning. Science, 299, 694-697. https://doi.org/10.1126/science.1078940

Eichwald, O., Ducasse, O., Merbahi, N., Yousfi, M., \& Dubois, D. (2006). Effect of order fluid models on flue gas streamer dynamics. Journal of Physics D: Applied Physics, 39, 99-107. https://doi.org/10.1088/0022-3727/39/1/015

Gallimberti, I., Hepworth, J. K., \& Klewe, R. C. (1974). Spectroscopic investigation of impulse corona discharges. Journal of Physics D: Applied Physics, 7, 880-898. https://doi.org/10.1088/0022-3727/7/6/315

Green, A. E. S., \& Peterson, L. R. (1968). Energy loss functions for electrons and protons in planetary gases. Journal of Geophysical Research, $73,233$.

Gurevich, V. V. (1961). On the theory of runaway electrons. Soviet Physics JETP, 12, 904

Gurevich, A. V., Milikh, G. M., \& Roussel-Dupre, R. (1992). Runaway electron mechanism of air breakdown and preconditioning during a thunderstorm. Physics Letters A, 165, 463-468. https://doi.org/10.1016/0375-9601(92)90348-P

Gurevich, A. V., \& Zybin, K. P. (2001). Reviews of topical problems: Runaway breakdown and electric discharges in thunderstorms. Physics Uspekhi, 44, 1119-1140. https://doi.org/10.1070/PU2001v044n11ABEH000939

Gurevich, A. V., Zybin, K. P., \& Medvedev, Y. V. (2007). Runaway breakdown in strong electric field as a source of terrestrial gamma flashes and gamma bursts in lightning leader steps. Physics Letters A, 361, 119-125. https://doi.org/10.1016/j.physleta.2006.05.063

Hagelaar, G. J. M., \& Pitchford, L. C. (2005). Solving the Boltzmann equation to obtain electron transport coefficients and rate coefficients for fluid models. Plasma Sources Science and Technology, 14, 722-733. https://doi.org/10.1088/0963-0252/14/4/011

Howard, J., Uman, M. A., Dwyer, J. R., Hill, D., Biagi, C., Saleh, Z., et al. (2008). Co-location of lightning leader X-ray and electric field change sources. Geophysical Research Letters, 35, L13817. https://doi.org/10.1029/2008GL034134

Ihaddadene, K. M. A. (2016). Numerical modeling of streamer disharges in preparation for the TARANIS space mission (Ph.D Thesis), University of Orleans, FR.

Ihaddadene, K. M. A., \& Celestin, S. J. (2014). Modeling of the increase of the electric field in the encounter between a negative and a positive streamer. AGU Fall Meeting Abstracts, AE33A-05.

Ihaddadene, M. A., \& Celestin, S. (2015). Increase of the electric field in head-on collisions between negative and positive streamers Geophysical Research Letters, 42, 5644-5651. https://doi.org/10.1002/2015GL064623

Ihaddadene, M. A., \& Celestin, S. (2017). Determination of sprite streamers altitude based on $\mathrm{N}_{2}$ spectroscopic analysis. Journal of Geophysical Research: Space Physics, 122, 1000-1014. https://doi.org/10.1002/2016JA023111

Kochkin, P., Köhn, C., Ebert, U., \& van Deursen, L. (2016). Analyzing -ray emissions from meter-scale negative discharges in ambient air. Plasma Sources Science and Technology, 25(4), 44002. https://doi.org/10.1088/0963-0252/25/4/044002

Kochkin, P., Lehtinen, N., van Deursen, A. L. P. J, \& Østgaard, N. (2016). Pilot system development in metre-scale laboratory discharge. Journal of Physics D: Applied Physics, 49, 425203. https://doi.org/10.1088/0022-3727/49/42/425203

Kochkin, P. O., Nguyen, C. V., van Deursen, A. P. J., \& Ebert, U. (2012). Experimental study of hard X-rays emitted from metre-scale positive discharges in air. Journal of Physics D: Applied Physics, 45, 425202. https://doi.org/10.1088/0022-3727/45/42/425202

Kochkin, P. O., van Deursen, A. P. J., \& Ebert, U. (2014). Experimental study of spatio-temporal development of metre-scale negative discharges in air. Journal of Physics D: Applied Physics, 47, 145203. https://doi.org/10.1088/0022-3727/47/14/145203

Kochkin, P. O., van Deursen, A. P. J., \& Ebert, U. (2015). Experimental study on hard X-rays emitted from metre-scale negative discharges in air. Journal of Physics D: Applied Physics, 48(2), 25205. https://doi.org/10.1088/0022-3727/48/2/025205

Köhn, C., Chanrion, O., Babich, L. P., \& Neubert, T. (2018). Streamer properties and associated X-rays in perturbed air. Plasma Sources Science and Technology, 27(1), 15017. https://doi.org/10.1088/1361-6595/aaa5d8

Köhn, C., Chanrion, O., \& Neubert, T. (2017). Electron acceleration during streamer collisions in air. Geophysical Research Letters, 44, 2604-2613. https://doi.org/10.1002/2016GL072216 
Köhn, C., Chanrion, O., \& Neubert, T. (2018). High energy emissions induced by air density fluctuations of discharges. Geophysical Research Letters, 45, 5194-5203. https://doi.org/10.1029/2018GL077788

Köhn, C., \& Ebert, U. (2015). Calculation of beams of positrons, neutrons, and protons associated with terrestrial gamma ray flashes. Journal of Geophysical Research: Atmospheres, 120, 1620-1635. https://doi.org/10.1002/2014JD022229

Kremnev, V. V., \& Kurbatov, Y. A. (1972). X-rays from a gas discharge in a strong electric field. Soviet Physics - Technical Physics, 17, 626.

Kulikovsky, A. A. (2000). The role of photoionization in positive streamer dynamics. Journal of Physics D: Applied Physics, 33, 1514-1524. https://doi.org/10.1088/0022-3727/33/12/314

Kulikovsky, A. A. (2001). Reply: Reply to comment on 'The role of photoionization in positive streamer dynamics'. Journal of Physics D: Applied Physics, 34, 251-252. https://doi.org/10.1088/0022-3727/34/2/16

Kulikovsky, A. A. (2002). Comment on "Spontaneous branching of anode-directed streamers between planar electrodes". Physical Review Letters, 89(22), 229401. https://doi.org/10.1103/PhysRevLett.89.229401

Landau, L. D., \& Lifshitz, E. M. (1960). Electrodynamics of continuous media (pp. 460). Moscow: Pergamon

Lefeuvre, F., Blanc, E., Pinçon, J.-L., Roussel-Dupré, R., Lawrence, D., Sauvaud, J.-A., et al. (2008). TARANIS-A satellite project dedicated to the physics of TLEs and TGFs. Space Science Reviews, 137, 301-315. https://doi.org/10.1007/s11214-008-9414-4

Lehtinen, N. G., Kochkin, P., \& Ostgaard, N. (2016). X-ray production in a laboratory streamer discharge. AGU Fall Meeting Abstracts, AE33A-0431.

Lehtinen, N., Ostgaard, N., \& Kochkin, P. (2016). Modeling of laboratory streamer discharge features leading to X-ray emissions. EGU General Assembly (Vol. 18, pp. EPSC2016-6180).

Li, C., Brok, W. J. M., Ebert, U., \& van der Mullen, J. J. A. M. (2007). Deviations from the local field approximation in negative streamer heads. Journal of Applied Physics, 101(12), 123,305-14. https://doi.org/10.1063/1.2748673

Li, C., Ebert, U., Brok, W. J. M., \& Hundsdorfer, W. (2008). Fast track communication: Spatial coupling of particle and fluid models for streamers: Where nonlocality matters. Journal of Physics D: Applied Physics, 41(3), 32005. https://doi.org/10.1088/0022-3727/41/3/ 032005

Li, C., Ebert, U., \& Hundsdorfer, W. (2009). Fast track communication: 3D hybrid computations for streamer discharges and production of runaway electrons. Journal of Physics D: Applied Physics, 42(20), 202003. https://doi.org/10.1088/0022-3727/42/20/202003

Litvinov, E. A., Mesyats, G. A., \& Proskurovskii, D. I. (1983). Reviews of topical problems: Field emission and explosive electron emission processes in vacuum discharges. Soviet Physics Uspekhi, 26, 265-302. https://doi.org/10.1070/PU1983v026n02ABEH004322

Liu, N., \& Pasko, V. P. (2004). Effects of photoionization on propagation and branching of positive and negative streamers in sprites. Journal of Geophysical Research, 109, A04301. https://doi.org/10.1029/2003JA010064

Liu, N., Pasko, V. P., Burkhardt, D. H., Frey, H. U., Mende, S. B., Su, H.-T., et al. (2006). Comparison of results from sprite streamer modeling with spectrophotometric measurements by ISUAL instrument on FORMOSAT-2 satellite. Geophysical Research Letters, 33, L01101. https://doi.org/10.1029/2005GL024243

Liu, N., Pasko, V. P., Frey, H. U., Mende, S. B., Su, H.-T., Chen, A. B., et al. (2009). Assessment of sprite initiating electric fields and quenching altitude of a ${ }^{1} \Pi_{g}$ state of $\mathrm{N}_{2}$ using sprite streamer modeling and ISUAL spectrophotometric measurements. Journal of Geophysical Research, 114, A00E02. https://doi.org/10.1029/2008JA013735

Luque, A. (2017). Radio frequency electromagnetic radiation from streamer collisions. Journal of Geophysical Research: Atmospheres, 122, 10,497-10,509. https://doi.org/10.1002/2017JD027157

March, V., \& Montanyà, J. (2011). X-rays from laboratory sparks in air: The role of the cathode in the production of runaway electrons Geophysical Research Letters, 38, L04803. https://doi.org/10.1029/2010GL046540

Mesyats, G. A., \& Bychkov, Y. I. (1968). Statistical study of nanosecond breakdown delay in narrow gas gaps in superhigh electric fields. Soviet Physics - Technical Physics, 12, 1258.

Mesyats, G. A., Bychkov, Y. I., \& Kremnev, V. V. (1972). Pulsed nanosecond electric discharges in air. Uspekhi Fizicheskikh Nauk, 107, 201-228.

Montanyà, J., Fabró, F., March, V., van der Velde, O., Solà, G., Romero, D., \& Argemí, O. (2015). X-rays and microwave RF power from high voltage laboratory sparks. Journal of Atmospheric and Solar-Terrestrial Physics, 136, 94-97. https://doi.org/10.1016/j.jastp.2015.06.009

Moore, C. B., Eack, K. B., Aulich, G. D., \& Rison, W. (2001). Energetic radiation associated with lightning stepped-leaders. Geophysical Research Letters, 28, 2141-2144.

Morrow, R., \& Lowke, J. J. (1997). Streamer propagation in air. Journal of Physics D: Applied Physics, 30, 614-627. https://doi.org/10.1088/ $0022-3727 / 30 / 4 / 017$

Moss, G. D., Pasko, V. P., Liu, N., \& Veronis, G. (2006). Monte Carlo model for analysis of thermal runaway electrons in streamer tips in transient luminous events and streamer zones of lightning leaders. Journal of Geophysical Research, 111, A02307. https://doi.org/10. 1029/2005ja011350

Naidis, G. V. (1996). On streamer interaction in a pulsed positive corona discharge. Journal of Physics D: Applied Physics, 29, 779-783. https://doi.org/10.1088/0022-3727/29/3/039

Naidis, G. V. (1997). Effects of nonlocality on the dynamics of streamers in positive corona discharges. Technical Physics Letters, 23, 493-494. https://doi.org/10.1134/1.1261717

Neubert, T. (2009). ASIM-An instrument suite for the International Space Station, AIP Conference Proceedings (Vol. 1118, pp. 8-12). https://doi.org/10.1063/1.3137718

Neubert, T., Østgaard, N., Reglero, V., Blanc, E., Chanrion, O., Oxborrow, C. A., et al. (2019). The ASIM mission on the international space mission. Space Science Reviews, 215, 26. https://doi.org/10.1007/s11214-019-0592-z

Nguyen, C. V., van Deursen, A. P. J., \& Ebert, U. (2008). Multiple X-ray bursts from long discharges in air. Journal of Physics D: Applied Physics, 41(23), 234012. https://doi.org/10.1088/0022-3727/41/23/234012

Nguyen, C. V., van Deursen, A. P. J., van Heesch, E. J. M., Winands, G. J. J., \& Pemen, A. J. M. (2010). X-ray emission in streamer-corona plasma. Journal of Physics D: Applied Physics, 43(2). https://doi.org/10.1088/0022-3727/43/2/025202

Nijdam, S., Moerman, J. S., Briels, T. M. P., van Veldhuizen, E. M., \& Ebert, U. (2008). Stereo-photography of streamers in air. Applied Physics Letters, 92(10), 101502. https://doi.org/10.1063/1.2894195

Østgaard, N., Carlson, B. E., Nisi, R. S., Gjesteland, T., Grøndahl, Ø., Skeltved, A., et al. (2016). Relativistic electrons from sparks in the laboratory. Journal of Geophysical Research: Atmospheres, 121, 2939-2954. https://doi.org/10.1002/2015JD024394

Pancheshnyi, S. V., \& Starikovskii, A. Y. (2001). Comment: Comments on 'The role of photoionization in positive streamer dynamics'. Journal of Physics D: Applied Physics, 34, 248-250. https://doi.org/10.1088/0022-3727/34/2/415

Papageorgiou, L., Metaxas, A. C., \& Georghiou, G. E. (2011). Three-dimensional numerical modelling of gas discharges at atmospheric pressure incorporating photoionization phenomena. Journal of Physics D: Applied Physics, 44(4), 45203. https://doi.org/10.1088/0022-3727/ $44 / 4 / 045203$ 
Pasko, V. P. (2006). Theoretical modeling of sprites and jets, Sprites, elves and intense lightning discharges, NATO science series II: Mathematics physics and chemistry (Vol. 225). Springer.

Peterson, L. R., \& Green, A. E. S. (1968). The relation between ionization yields, cross sections and loss functions. Journal of Physics B: Atomic and Molecular Physics, $1,1131$.

Plewa, J. M., Eichwald, O., Ducasse, O., Dessante, P., Jacobs, C., Renon, N., \& Yousfi, M. (2018). 3D streamers simulation in a pin to plane configuration using massively parallel computing. Journal of Physics D: Applied Physics, 51, 95206. https://doi.org/10.1088/1361-6463/ aaa91b

Qin, J. (2013). Numerical modeling of the inception morphology and radio signals of sprites produced by lightning discharges with positive and negative polarity (Ph.D. thesis), The Pennsylvania State University.

Qin, J., \& Pasko, V. P. (2014). On the propagation of streamers in electrical discharges. Journal of Physics D: Applied Physics, 47(435202). https://doi.org/10.1088/0022-3727/47/43/435202

Rahman, M., Cooray, V., Azlinda Ahmad, N., Nyberg, J., Rakov, V. A., \& Sharma, S. (2008). X rays from 80-cm long sparks in air. Geophysical Research Letters, 35, L06805. https://doi.org/10.1029/2007GL032678

Raizer, Y. P. (1991). Gas discharge physics (pp. 449). Berlin Heidelberg: Springer.

Rakov, V. A., \& Raizer, Y. P. (2000). Lightning physics and lightning protection (pp. 321). London: IOP.

Reess, T., Ortega, P., Gibert, A., Domens, P., \& Pignolet, P. (1995). An experimental study of negative discharge in $1.3 \mathrm{~m}$ point-plane air gap: The function of the space stem in the propagation mechanism. Journal of Physics D: Applied Physics, 28, 2306-2313.

Rocco, A., Ebert, U., \& Hundsdorfer, W. (2002). Branching of negative streamers in free flight. Physical Review E, 66(3), 35102. https://doi. org/10.1103/PhysRevE.66.035102

Saleh, Z., Dwyer, J., Howard, J., Uman, M., Bakhtiari, M., Concha, D., et al. (2009). Properties of the X-ray emission from rocket-triggered lightning as measured by the Thunderstorm Energetic Radiation Array (TERA). Journal of Geophysical Research, 114, D17210. https:// doi.org/10.1029/2008JD011618

Schaal, M. M., Dwyer, J. R., Arabshahi, S., Cramer, E. S., Lucia, R. J., Liu, N. Y., et al. (2014). The structure of X-ray emissions from triggered lightning leaders measured by a pinhole-type X-ray camera. Journal of Geophysical Research: Atmospheres, 119, 982-1002. https://doi. org/10.1002/2013JD020266

Shi, F., Liu, N., \& Dwyer, J. (2017). Three-dimensional modeling of two interacting streamers. Journal of Geophysical Research: Atmospheres, 122, 10,169-10,176. https://doi.org/10.1002/2017JD026935

Shi, F., Liu, N., \& Dwyer, J. (2018). Field enhancement and radio emissions from head-on collisions of streamers. URSI Fall Meeting Abstracts, HEG1-3.

Shi, F., Liu, N., Dwyer, J., \& Ihaddadene, K. M. A. (2019). VHF and UHF electromagnetic radiation produced by streamers in lighning. Geophysical Research Letters, 46, 443-451. https://doi.org/10.1029/2018GL080309

Stankevich, Y. L. (1971). Initial stage of an electrical discharge in a dense gas. Soviet Physics - Technical Physics, $15,1138$.

Stankevich, Y. L., \& Kalinin, V. G. (1968). Fast electrons and X-ray radiation during the initial stage growth of a pulsed spark discharge in air. Soviet Physics - Doklady, 20, 1042

Stratton, J. A. (1941). Electromagnetic theory (pp. 615). York: Maple.

Tarasova, L. V., \& Khudyakova, L. N. (1970). X-rays from pulsed discharges in air. Soviet Physics - Technical Physics, 14, 1048.

Teunissen, J., \& Ebert, U. (2017). Simulating streamer discharges in 3D with the parallel adaptive Afivo framework. Journal of Physics D: Applied Physics, 50, 474001. https://doi.org/10.1088/1361-6463/aa8faf

Zubarev, N. M., Mesyats, G. A., \& Yalandin, M. I. (2017). Conditions for runaway electrons in a gas diode with a strongly nonuniform electric field. Journal of Experimental and Theoretical Physics Letters, 105, 537-541. https://doi.org/10.1134/S002136401708015X 